\title{
THE GRADED WITT RING AND GALOIS COHOMOLOGY. II
}

\author{
JÓN KR. ARASON, RICHARD ELMAN, AND BILL JACOB
}

\begin{abstract}
A primary problem in the theory of quadratic forms over a field $F$ of characteristic different from two is to prove that the rings $H_{q}^{*} F$ and $G W F$ are isomorphic. Here $\left.H_{q}^{*} F=H^{*}\left(\mathrm{Gal}\left(F_{q} / F\right), \mathbf{Z} / 2 \mathbf{Z}\right)\right)$, where $F_{q}$ is the quadratic closure of $F$, and $G W F$ is the graded Witt ring associated to the fundamental ideal of even dimensional forms in the Witt ring $W F$ of $F$. In this paper, we assume we are given a field extension $K$ of $F$ such that $W K$ is 'close' to $W F$ or $H_{q}^{*} K$ is 'close' to $H_{q}^{*} F$. A method is developed to obtain information about these graded rings over $F$ and its 2-extensions from information about the corresponding graded ring of $K$. This relative theory extends and includes the previously developed absolute case where $K=F_{q}$. Applications are also given to show that $H_{q}^{*} F$ and $G W F$ are isomorphic for a collection of fields arising naturally from the theory of abstract Witt rings.
\end{abstract}

\section{INTRODUCTION}

The basic motivation for this paper is the interaction between the algebraic theory of quadratic forms over fields and Galois cohomology.

Throughout $F$ will be an arbitrary field of characteristic different from two. We denote by $W F$ the Witt ring of equivalence classes of quadratic forms over $F$ and by $I F$ the ideal of even dimensional forms in $W F$. (Cf. [L] or [Sch] for terminology from the theory of quadratic forms.) We write $I^{n} F$ for the $n$th power of $I F$ and denote by $G W F$ the associated graded ring $\bigoplus_{n=0}^{\infty} I^{n} F / I^{n+1} F$. We call $G W F$ the graded Witt ring of $F$.

Let $H^{*} F:=H^{*}(F, \mathbf{Z} / 2 \mathrm{Z})$ be the ('full') $\bmod 2$ cohomology ring of $F$, i.e., the cohomology ring $H^{*}\left(G_{F}, \mathrm{Z} / 2 \mathrm{Z}\right)$ where $G_{F}$ is the absolute Galois group of $F$. (Cf. [S] for terminology from Galois cohomology.) By [A1, Satz 4.8 or EL1, Main Theorem 3.2] the assignment $\left\langle 1,-a_{1}\right\rangle \otimes \cdots \otimes\left\langle 1,-a_{n}\right\rangle \mapsto\left(a_{1}\right) \cup \cdots \cup\left(a_{n}\right)$ defines a map from the set of $n$-fold Pfister forms in $W F$ to the set of $n$ fold cup products in $H^{*} F$. Here $(a)$ in $H^{1} F \cong \dot{F} / \dot{F}^{2}$ denotes the element corresponding to the square class of $a$ in $\dot{F}:=F \backslash\{0\}$. Our first main question is whether this map extends to a homomorphism $e_{F}^{n}: I^{n} F \rightarrow H^{n} F$. We note that $I^{n} F$ is, even as a group, generated by the $n$-fold Pfister forms in $W F$, so the question is not how to define $e_{F}^{n}$ but whether $e_{F}^{n}$ is well-defined. If $e_{F}^{n}$ is

Received by the editors February 8, 1988.

1980 Mathematics Subject Classification (1985 Revision). Primary 11E04, 11E81, 12G05; Secondary $19 \mathrm{G} 12$.

Supported in part by the National Science Foundation. 
well-defined then its kernel contains $I^{n+1} F$, hence it induces a homomorphism $\bar{e}_{F}^{n}: I^{n} F / I^{n+1} F \rightarrow H^{n} F$. Our second main question is then whether $\bar{e}_{F}^{n}$ is an isomorphism. Note that if both questions have an affirmative answer for all $n$ then the resulting isomorphism $\bar{e}_{F}^{*}: G W F \rightarrow H^{*} F$ is an isomorphism of graded rings.

If $n$ is small then the answers are positive. The homomorphism $e_{F}^{0}$ is the dimension index and $\bar{e}_{F}^{0}$ is trivially an isomorphism. The homomorphism $e_{F}^{1}$ is the (signed) discriminant and Pfister showed $\bar{e}_{F}^{1}$ to be an isomorphism in [P, Korollar to Satz 13]. The homomorphism $e_{F}^{2}$ is the classical Clifford invariant and $\bar{e}_{F}^{2}$ was shown to be an isomorphism by Merkurjev (cf. [Me]). The homomorphism $e_{F}^{3}$ was shown to be well-defined by the first author (cf. [A1, Satz 5.7]) and $\bar{e}_{F}^{3}$ was shown to be an isomorphism by Merkurjev-SuslinRost (cf. [MS, R]). Recently, the map $e_{F}^{4}$ was shown to be well-defined by the third author and Rost (cf. [JR]).

One would, a priori, only expect the theory of quadratic forms over $F$ to give information about 2-extensions of $F$, viz., about the Galois group $\operatorname{Gal}\left(F_{q} / F\right)$. Here, and throughout, we denote by $F_{q}$ the quadratic closure (i.e., 2-closure) of $F$. From the viewpoint of quadratic forms it is, therefore, more natural to ask about the relationship between $G W F$ and the quadratic mod 2 cohomology ring $\left.H_{q}^{*} F:=H^{*}\left(\operatorname{Gal}\left(F_{q} / F\right), \mathrm{Z} / 2 \mathrm{Z}\right)\right)$. As before we have a map $\left\langle 1,-a_{1}\right\rangle \otimes$ $\cdots \otimes\left\langle 1,-a_{n}\right\rangle \mapsto\left(a_{1}\right) \cup \cdots \cup\left(a_{n}\right)$ from the set of $n$-fold Pfister forms in $W F$ to the set of $n$-fold cup products in $H_{q}^{*} F$. This time we view $(a)$ as an element in $H_{q}^{1} F \cong \dot{F} / \dot{F}^{2}$. Hence we also have the question whether this map extends to a homomorphism $e_{q, F}^{n}: I^{n} F \rightarrow H_{q}^{n} F$ and, if it does, whether the induced map $\bar{e}_{q, F}^{n}: I^{n} F / I^{n+1} F \rightarrow H_{q}^{n} F$ is an isomorphism.

Of course, the two sets of problems are closely related. Indeed it is conjectured that the inflation map inf: $H_{q}^{*} F \rightarrow H^{*} F$ is an isomorphism, making both sets of problems equivalent. This conjecture is equivalent to $H^{n} F_{q}=0$ for all $n>0$, i.e., to $\bar{e}_{F_{q}}^{*}$ being an isomorphism. In particular, if $\bar{e}_{F}^{*}: G W F \rightarrow H^{*} F$ is a well-defined isomorphism for every field $F$ then also $\bar{e}_{q, F}^{*}: G W F \rightarrow H_{q}^{*} F$ is a well-defined isomorphism for every field $F$. The converse is also true (cf. [AEJ1, Proposition 5.9]). The general results on the $e_{F}^{n}$ mentioned above imply that $H^{n} F_{q}=0$ for $0<n<4$. Hence inf: $H_{q}^{n} F \rightarrow H^{n} F$ is an isomorphism for $0<n<4$ and is injective for $n=4$. In particular, the corresponding results also hold for the $e_{q, F}^{n}$.

In this paper, we take the latter viewpoint. In particular, we want to find conditions on $F$ such that $\bar{e}_{q, F}^{*}: G W F \rightarrow H_{q}^{*} F$ is a well-defined isomorphism.

In our papers [AEJ1 and AEJ3], we answered our questions in the affirmative for fields $F$ such that $I^{4} F$ or $H_{q}^{4} F$ is "small". For example, we proved that $\bar{e}_{q, F}^{*}$ is a well-defined isomorphism whenever $I^{4} F=0$ or $H_{q}^{4} F=0$. Under 
suitable hypotheses on the $e_{q, F}^{m}$ for small $m$, the methods and proofs in these papers immediately give more general results. We now state some of these.

Theorem 1.1. Suppose that $\bar{e}_{q, M}^{m}$ is a well-defined isomorphism for all $m<n$ and $e_{q, M}^{n}$ is well-defined for every finite 2-extension $M$ of $F$. Then $I^{n+1} F=0$ or $I^{n+1} F(\sqrt{-1})=0$ implies that $\bar{e}_{q, F}^{*}$ is a well-defined isomorphism.

Proof. Cf. [AEJ1, Theorem 3.7 and Theorem 5.19].

In Theorem 1.1 replacing 'every finite 2-extension' by 'every finite extension' and every $e_{q}$ by $e$ also results in a valid theorem.

In connection with the conclusion of the theorem, we note that $I^{n+1} F=2 I^{n} F$ and $I^{n+1} F$ is torsion-free implies that $I^{n+1} F(\sqrt{-1})=0$ (cf. [EP, Theorem 3.3]). Here $2 I^{n} F:=\langle 1,1\rangle \otimes I^{n} F$. The converse is, however, not known. Therefore, the following corollary is of interest.

Corollary 1.2. Suppose that $\bar{e}_{q, M}^{m}$ is a well-defined isomorphism for all $m<n$ and $e_{q, M}^{n}$ is well-defined for every finite 2-extension $M$ of $F$. Then $I^{n+1} F(\sqrt{-1})$ $=0$ if and only if $I^{n+1} F=2 I^{n} F$ and $I^{n+1} F$ is torsion-free.

Proof. Cf. [AEJ1, Theorem 5.19].

It follows immediately from the theorem, assuming its hypotheses, that $I^{n+1} F$ $=0$ implies that $H_{q}^{n+1} F=0$. For this, we have a converse but with a slightly stronger hypothesis. More generally, we have the following

Theorem 1.3. Suppose that $\bar{e}_{q, M}^{m}$ is a well-defined isomorphism for all $m<n$ and $e_{q, M}^{n}$ is well-defined for every field extension $M$ of $F$. Then two $(n+1)$ fold Pfister forms over $F$ are isometric if and only if their associated cohomology classes in $H_{q}^{n+1} F$ are equal.

Proof. Cf. [AEJ3, Theorem 1]. Note that in the double equality on line 6 on p. 652 in [AEJ3] only the inclusion $\operatorname{ker}\left(e_{q, F}^{n}\right) \subseteq J_{n+1} F$ is needed.

Corollary 1.4. Suppose that $\bar{e}_{q, M}^{m}$ is a well-defined isomorphism for all $m<n$ and $e_{q, M}^{n}$ is well defined for every field extension $M$ of $F$. Then $I^{n+1} F=0$ if and only if $H_{q}^{n+1} F=0$.

Combining Corollary 1.4 and Theorem 1.1, we get the following

Theorem 1.5. Suppose that $\bar{e}_{q, M}^{m}$ is a well-defined isomorphism for all $m<n$ and $e_{q, M}^{n}$ is well-defined for every field extension $M$ of $F$. Then $H^{n+1} F=0$ or $H^{n+1} F(\sqrt{-1})=0$ implies that $\bar{e}_{q, F}^{*}$ is a well-defined isomorphism.

By the results cited above these theorems apply for $n=4$. In particular, if the cohomological 2-dimension of $F(\sqrt{-1})$ is at most four then $\bar{e}_{q, F}^{*}$ is a well-defined isomorphism. This includes fields of transcendence degree at most four over a real closed or an algebraically closed field, fields of transcendence 
degree at most three over a finite field, and fields of transcendence degree at most two over a local or global field. Using the following theorem, we see that the same holds if $F$ is a $C_{5}$-field. This includes fields of transcendence degree five over an algebraically closed field and fields of transcendence degree four over a finite field.

Theorem 1.6. Suppose that $\bar{e}_{q, M}^{m}$ is a well-defined isomorphism for all $m<n$ and $e_{q, M}^{n}$ is well-defined for every field extension $M$ of $F$. If every anisotropic form in $I^{n} F$ has dimension less than or equal to $2^{n+1}$ then $\bar{e}_{q, F}^{*}$ is a well-defined isomorphism.

Proof. Cf. [AEJ3, Proposition 2].

Thus there is increasing evidence for the conjecture that $\bar{e}_{q, F}^{*}$ is a well-defined isomorphism for every field $F$. (The appropriate analogs of $(1.3)-(1.6)$ for the $e_{F}^{n}$ are also true (cf. [AEJ1 and $\left.\left.\mathrm{AEJ} 3\right]\right)$.)

In this paper, we study consequences of 'relative' versions of the conditions in Theorem 1.1 and Theorem 1.5. This means that we work with a field extension $K$ of $F$ and assume, instead of $I^{n+1} F$ being 'small', that $I^{n+1} F$ is 'close to' $I^{n+1} K$ or, instead of $H_{q}^{n+1} F$ being 'small', that $H_{q}^{n+1} F$ is 'close to' $H_{q}^{n+1} K$. If $K=F_{q}$ then the 'relative' conditions are equivalent to the 'absolute' conditions used in Theorem 1.1 and Theorem 1.5. For example, our 'relative' counterpart of the condition $H_{q}^{n+1} F=0$ will be that the natural morphism $H_{q}^{n} F \rightarrow H_{q}^{n} K$ is surjective and the natural morphism $H_{q}^{n+1} F \rightarrow H_{q}^{n+1} K$ is injective. (It follows, as we shall see in Theorem 3.9 below, that $H_{q}^{m} F \rightarrow H_{q}^{m} K$ is an isomorphism for every $m>n$.) We express this condition by saying that $K$ is $n$-taut over $F$. In $\S 3$, we study $n$-tautness and a weaker condition better suited to the case when $F$ is formally real. (When $K=F_{q}$, this weaker condition is equivalent to $H^{n+1} F(\sqrt{-1})=0$.) In $\S 4$, we introduce and study corresponding conditions, called $n$-tightness, for the theory of quadratic forms. In $\S 5$, we prove a 'relative' version of Theorem 1.1 in a slightly weaker form. In the last section, we present an application of the machinery developed in the preceding sections. We show that if $W F$ can be built up from 'small' abstract Witt rings, using certain natural constructions in the category of abstract Witt rings, then $\bar{e}_{q, E}^{*}$ is a well-defined isomorphism for every 2-extension $E$ of $F$. This includes the case when $W F$ is an elementary Witt ring (cf. [Ma]). In particular, the results in [AEJ2] are subsumed by the results here.

\section{CoMmutative SEMisimple AlgEBRAS}

The idea behind the results of this paper is to obtain information about $e_{q, F}^{n}$ from knowledge about $e_{q, K}^{n}$ where $K$ is a field extension of $F$ such that $i_{K / F}: W F \rightarrow W K$ is 'not too far from being an isomorphism'. It is, however, not only more general, but also more convenient, to consider arbitrary commutative semisimple $F$-algebras $K$. To do that we need some preparation. 
In what follows every commutative semisimple ring will have 2 a unit. Such a ring $K$ is canonically isomorphic to a direct product $\prod_{i} K_{i}$ of finitely many fields $K_{i}$. (Indeed $K \cong \prod_{\mathscr{P}} K / \mathscr{P}$ where $\mathscr{P}$ runs through the prime ideals of $K$.) We define

$$
H_{q}^{*} K:=\prod_{i} H_{q}^{*} K_{i} \quad \text { and } \quad W K:=\prod_{i} W K_{i} .
$$

Using the corresponding facts for fields, it is easy to see that $H_{q}^{*} K$ and $W K$ behave functorially in $K$. (In fact, our definition of $W K$ is consistent with that of Knebusch [K], which gives a functor on the category of schemes. Also $H_{q}^{*} K$ is a "2-etale" cohomology ring, defined functorially for all schemes.) Of course, we also let

$$
I K:=\prod_{i} I K_{i} \quad \text { so that } I^{n} K=\prod_{i} I^{n} K_{i} .
$$

It is then clear what we mean by $e_{q, K}^{n}$ being well-defined, etc.

Let $L$ be a commutative semisimple extension of a commutative semisimple ring $K$ that is free of rank 2 over $K$. Then $L$ is necessarily of the form $K \oplus K x$ such that $x^{2}$ is a unit $d$ in $K$. Clearly, the isomorphism class of $L$ over $K$ only depends on the class of $d$ modulo the group of squares of units in $K$. Conversely, this square class of $d$ is determined by the isomorphism class of $L$ over $K$. If $K=\prod_{i} K_{i}$ is a decomposition of $K$ into a direct product of fields $K_{i}$ and $d_{i} \in \dot{K}_{i}$ is the $i$ th coordinate of $d$ then $L=\prod_{i} L_{i}$, where $L_{i}=K_{i} \oplus K_{i} x_{i}$ with $x_{i}^{2}=d_{i}$. If $d_{i}$ is not a square in $K_{i}$ then $L_{i}$ is a quadratic field extension of $K_{i}$, and we have the exact triangle of cohomology

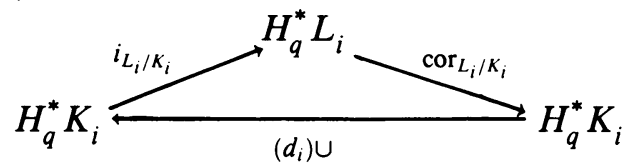

where $i_{L_{i} / K_{i}}$ is the ordinary restriction map in group cohomology and $\operatorname{cor}_{L_{i} / K_{i}}$ is the ordinary corestriction map in group cohomology (cf. [A1, Satz 4.5]). If $d_{i}=a_{i}^{2}$ is a square in $K_{i}$ then $L_{i}$ has orthogonal idempotents $\left(a_{i}+x_{i}\right) / 2 a_{i}$ and $\left(a_{i}-x_{i}\right) / 2 a_{i}$. So $L_{i} \cong K_{i} \times K_{i}$ with the inclusion $K_{i} \subseteq L_{i}$ corresponding to the diagonal mapping and with $x_{i}$ corresponding to $\left(a_{i},-a_{i}\right)$. The induced map

$$
i_{L_{i} / K_{i}}: H_{q}^{n} K_{i} \rightarrow H_{q}^{n} L_{i} \cong H_{q}^{n} K_{i} \times H_{q}^{n} K_{i}
$$

is, of course, the diagonal mapping. By defining

$$
\operatorname{cor}_{L_{i} / K_{i}}: H_{q}^{n} L_{i} \cong H_{q}^{n} K_{i} \times H_{q}^{n} K_{i} \rightarrow H_{q}^{n} L_{i}
$$

to be the codiagonal mapping, we get a short exact sequence

$$
0 \rightarrow H_{q}^{n} K_{i} \rightarrow H_{q}^{n} L_{i} \rightarrow H_{q}^{n} K_{i} \rightarrow 0
$$

for each $n$. But, as $d_{i}$ is a square in $K_{i}$, cup product with $\left(d_{i}\right)$ is trivial, so that these short exact sequences fit into an exact triangle of cohomology just as above. The direct product of all these exact triangles is also an exact triangle. Thus we have the following proposition. 
Proposition 2.1. Let $L / K$ be an extension of commutative semisimple rings such that $L$ is free of rank 2 over $K$. Write $L=K \oplus K x$ with $x^{2}=d$ where $d$ is a unit in $K$. Then we have the exact triangle of cohomology:

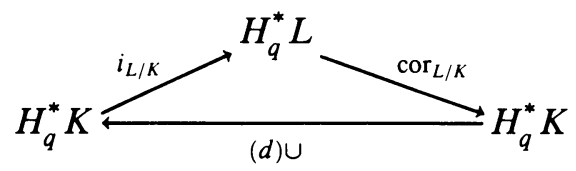

This exact triangle unwraps into a long exact sequence

$$
\begin{aligned}
0 & \rightarrow H_{q}^{0} K \rightarrow H_{q}^{0} L \rightarrow H_{q}^{0} K \rightarrow H_{q}^{1} K \rightarrow H_{q}^{1} L \\
& \rightarrow H_{q}^{1} K \rightarrow H_{q}^{2} K \rightarrow H_{q}^{2} L \rightarrow H_{q}^{2} K \rightarrow \cdots .
\end{aligned}
$$

The exact triangle of cohomology is functorial in $K$. More precisely, we have the following

Proposition 2.2. Let $L / K$ be an extension of commutative semisimple rings such that $L$ is free of rank 2 over $K$. Write $L=K \oplus K x$ with $x^{2}=d$ where $d$ is $a$ unit in $K$. Let $M$ be a commutative semisimple ring and $f: K \rightarrow M$ a ring homomorphism. Let $e:=f(d)$ and let $N:=M \oplus M y$ with $y^{2}=e$. Denote by $g$ the ring homomorphism $L \rightarrow N$ extending $f$ with $g(x)=y$. Then the following diagram is commutative:

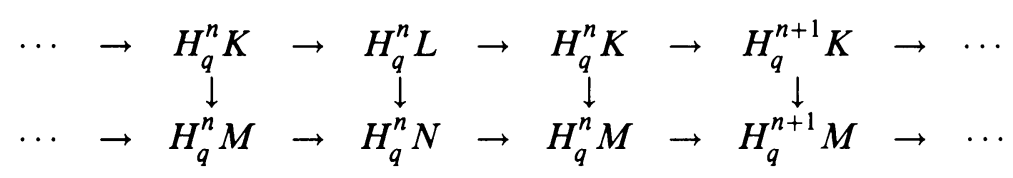

Here the horizontal arrows are the mappings in the sequence $(\Delta)$ and the vertical arrows are the homomorphisms induced by $f$ and $g$.

Proof. Only the commutativity of the middle square is not a priori clear. In proving that this square is also commutative, one easily reduces to the case where $K$ and $M$ are both fields. We then may assume that $K \subseteq M$ and $f$ is the inclusion. The case where $L$ and $N$ are also both fields is known (cf. [A1, Satz 4.5]). If $L$ is not a field then $N$ is also not a field. In this case, the commutativity follows at once from our definition of cor. There remains the case where $L$ is a field but $N$ is not a field, i.e., where $d$ is not a square in $K$ but is a square in $M$, say $d=c^{2}$. By mapping $x(=\sqrt{d})$ onto $c$, we embed $L$ in $M$. As previously remarked, we then have $N \cong M \times M$ such that the inclusion $M \subseteq N$ takes $c$ to $(c,-c)$. Consequently, the composition $L \rightarrow$ $N \cong M \times M$ is given by $w \mapsto(w, \bar{w})$, where the bar denotes the nontrivial $K$ automorphism of $L$. It follows that the induced homomorphism $i_{N / L}: H_{q}^{n} L \rightarrow$ $H_{q}^{n} N \cong H_{q}^{n} M \times H_{q}^{n} M$ is given by $\gamma \mapsto\left(i_{M / L}(\gamma), i_{M / L}(\bar{\gamma})\right)$, where we also use the bar to denote the automorphism induced on $H_{q}^{n} L$. By the definition of $\operatorname{cor}_{N / M}$, we have

$$
\operatorname{cor}_{N / M}\left(i_{M / L}(\gamma)\right)=i_{M / L}(\gamma)+i_{M / L}(\bar{\gamma})=i_{M / L}(\gamma+\bar{\gamma})
$$


for every $\gamma$ in $H_{q}^{n} L$. On the other hand, cor $_{L / K}$ is the usual corestriction and $i_{L / K}$ the restriction, so that (cf., for example, [Br, Proposition III.9.5]) we have $i_{L / K}\left(\operatorname{cor}_{L / K}(\gamma)\right)=\gamma+\bar{\gamma}$. It follows that

$$
i_{M / K}\left(\operatorname{cor}_{L / K}(\gamma)\right)=i_{M / L}\left(i_{L / K}\left(\operatorname{cor}_{L / K}(\gamma)\right)\right)=i_{M / L}(\gamma+\bar{\gamma})
$$

for every $\gamma$ in $H_{q}^{n} L$.

For the Witt ring, we have analogous results.

Proposition 2.3. Let $L / K$ be an extension of commutative semisimple rings such that $L$ is free of rank 2 over $K$. Write $L=K \oplus K x$ with $x^{2}=d$ where $d$ is $a$ unit in $K$. Then we have the exact triangle of Witt rings:

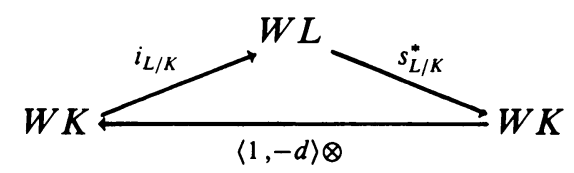

Here the morphism $s_{L / K}^{*}$ is induced by the $K$-linear map $s_{L / K}: L \rightarrow K$ determined by $1 \mapsto 0$ and $x \mapsto 1$. This exact triangle unwraps into a long zero sequence

$(\nabla) W K \rightarrow W L \rightarrow W K \rightarrow I^{1} K \rightarrow I^{1} L \rightarrow I^{1} K \rightarrow I^{2} K \rightarrow I^{2} L \rightarrow I^{2} K \rightarrow \cdots$.

Proof. The proof is analogous to the proof of Proposition 2.1. For the case $d_{i}$ is not a square in $K_{i}$ cf. [A1, Satz 2.4 or EL3, Theorem 2.6]. When $d_{i}$ $=a_{i}^{2}$, one uses that the $K_{i}$-linear map $s_{L_{i} / K_{i}}: L_{i} \rightarrow K_{i}$ maps $\left(a_{i} \pm x_{i}\right) / 2 a_{i} \mapsto$ $\pm 1 / 2 a_{i}$. It follows that $s_{L_{i} / K_{i}}^{*}: W L_{i} \cong W K_{i} \times W K_{i} \rightarrow W K_{i}$ maps $\left(\phi_{1}, \phi_{2}\right) \mapsto$ $\left(\phi_{1}-\phi_{2}\right) / 2 a_{i}$.

Note that $s_{L / K}^{*}$ depends on the choice of $x$. Hence we have to be careful in the formulation of the "functorality".

Proposition 2.4. Let $L / K$ be an extension of commutative semisimple rings such that $L$ is free of rank 2 over $K$. Write $L=K \oplus K x$ with $x^{2}=d$ where $d$ is a unit in $K$. Let $M$ be a commutative semisimple ring and $f: K \rightarrow M$ be a ring homomorphism. Let $e:=f(d)$ and let $N:=M \oplus M y$ with $y^{2}=e$. Denote by $g$ the ring homomorphism $L \rightarrow N$ extending $f$ with $g(x)=y$. Let $s_{L / K}^{*}: W L \rightarrow W K$ be the morphism induced by the $K$-linear map $s_{L / K}: L \rightarrow K$ determined by $1 \mapsto 0$ and $x \mapsto 1$ and let $s_{M / N}^{*}: W N \rightarrow W M$ be the morphism induced by the $M$-linear map $s_{M / N}: N \rightarrow M$ determined by $1 \mapsto 0$ and $y \mapsto 1$. Then the following diagram is commutative.

$$
\begin{aligned}
& \cdots \rightarrow I^{n} K \rightarrow I^{n} L \rightarrow I^{n} K \rightarrow I^{n+1} K \rightarrow \cdots \\
& \cdots \stackrel{\downarrow}{\stackrel{\downarrow}{ }} \rightarrow \stackrel{\downarrow}{I^{n} M} \rightarrow I^{n} N \rightarrow I^{n} M \rightarrow I^{n+1} M \rightarrow \cdots
\end{aligned}
$$

Here the horizontal arrows are the mappings in the zero sequence $(\nabla)$ and the vertical arrows are the homomorphisms induced by $f$ and $g$. 
Proof. This is clear. Of course, for the commutativity at the middle square one has to use that $s_{M / N} \circ g=f \circ s_{L / K}$.

It is not known if the zero sequence $(\nabla)$ is always exact. Indeed, one of the main challenges in the algebraic theory of quadratic forms is to prove that this sequence is always exact. However, we are able to show exactness in this sequence in cases where we can use the exact sequence $(\Delta)$ of cohomology. For example, we have the following

Proposition 2.5. Let $L / K$ be an extension of commutative semisimple rings such that $L$ is free of rank 2 over $K$. Write $L=K \oplus K x$ with $x^{2}=d$ where $d$ is a unit in $K$. Assume that $\bar{e}_{q, K}^{m}$ and $\bar{e}_{q, L}^{m}$ are well-defined isomorphisms for all $m<n$. Then the sequence

$$
\cdots \rightarrow I^{n-1} K \rightarrow I^{n} K \rightarrow I^{n} L \rightarrow I^{n} K \rightarrow I^{n+1} K
$$

is exact.

Proof. This is done by induction on $n$. The commutative diagram

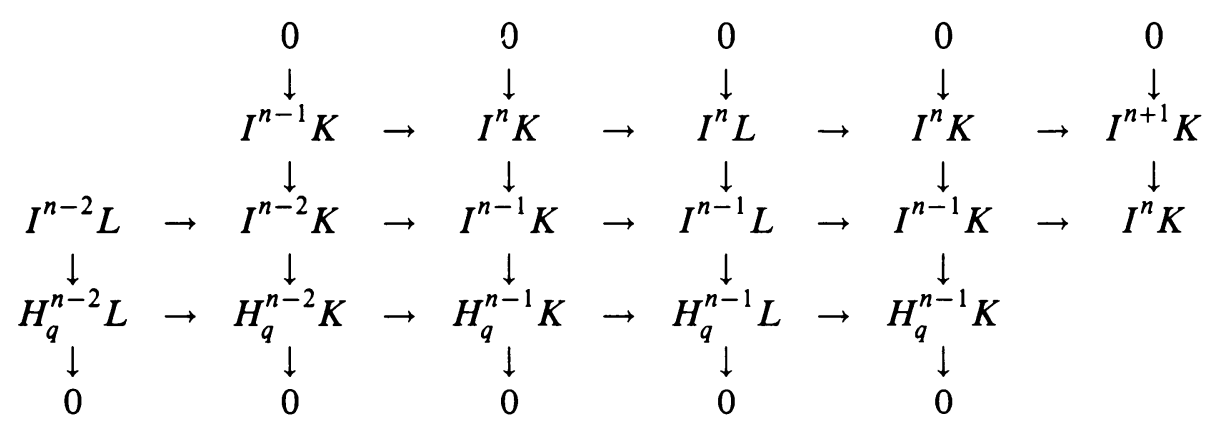

has exact columns by hypothesis. The bottom row is exact by Proposition 2.1 and the middle row is exact by the induction hypothesis. One chases in this diagram to establish the induction step.

In the case we have, in addition to the hypothesis of the proposition, that $e_{q, K}^{n}$ and $e_{q, L}^{n}$ are also well-defined, we can extend the exact sequence. However, we have to use kernels of $e_{q}^{n}$ instead of $I^{n+1}$. To simplify the statement, we fix the following notation: If $e_{q, K}^{n}$ is well-defined we let

$$
J_{n+1} K:=\operatorname{ker}\left(e_{q, K}^{n}\right) .
$$

Clearly, $J_{n+1} K$ is an ideal in $W K$ such that $I^{n+1} K \subseteq J_{n+1} K \subseteq I^{n} K$. Of course, $\bar{e}_{q, K}^{n}$ is injective if and only if $J_{n+1} K=I^{n+1} K$.

We do not know if our ideals $J_{n+1}$ are always the same as the ideals $J_{n+1}$ of [K]. However, for $n \leq 4$ they do agree. (For $n \leq 2 \mathrm{cf}$. [K, Example 6.2], for $n=3 \mathrm{cf}$. [A3, Proposition 3], and for $n=4$ it follows as in [A3, Proposition 3] using [MS or R and JR].) 
Proposition 2.6. Let $L / K$ be an extension of commutative semisimple rings such that $L$ is free of rank 2 over $K$. Write $L=K \oplus K x$ with $x^{2}=d$ where $d$ is a unit in $K$. Assume that $\bar{e}_{q, K}^{m}$ and $\bar{e}_{q, L}^{m}$ are well-defined isomorphisms for all $m<n$ and that $e_{q, K}^{n}$ and $e_{q, L}^{n, K}$ are well-defined. Then the sequence

$$
I^{n} K \rightarrow I^{n} L \rightarrow I^{n} K \rightarrow J_{n+1} K \rightarrow J_{n+1} L \rightarrow J_{n+1} K
$$

is exact.

Proof. We only have to prove exactness at $J_{n+1} K$ and $J_{n+1} L$. That is done by chasing in the commutative diagram

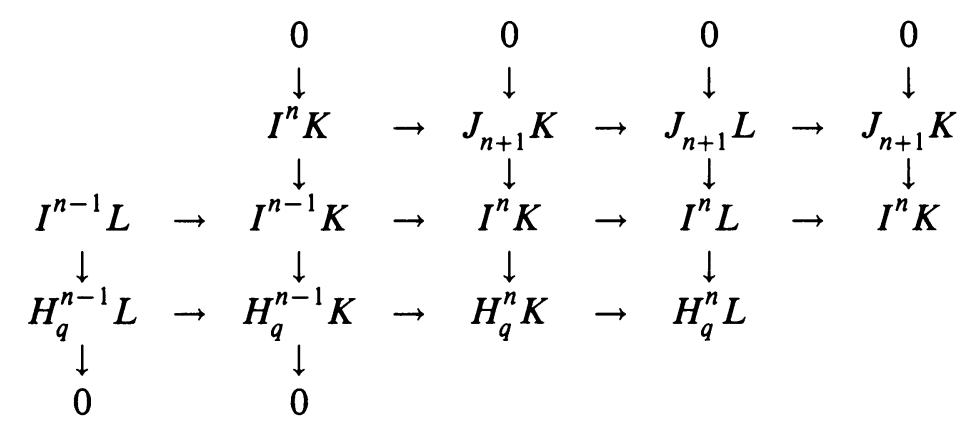

as in the proof of Proposition 2.5.

The exactness of the sequence in the conclusion has immediate consequences regarding the injectivity of $\bar{e}_{q, F}^{n}$. We shall need the following in $\S 5$.

Proposition 2.7. Let $L / K$ be an extension of commutative semisimple rings such that $L$ is free of rank 2 over $K$. Write $L=K \oplus K x$ with $x^{2}=d$ where $d$ is a unit in $K$. Assume that $e_{q, K}^{n}$ and $e_{q, L}^{n}$ are well-defined and that the sequence

$$
I^{n} K \rightarrow J_{n+1} K \rightarrow J_{n+1} L
$$

is exact. Then $\bar{e}_{q, F}^{n}$ is injective on the image of $\langle 1,-d\rangle \otimes I^{n-1} K$ in $I^{n} K / I^{n+1} K$. Proof. Clear.

\section{TAUT ALGEBRAS}

Let $K$ be a commutative semisimple $F$-algebra. We view the embedding $F \rightarrow K$ as an inclusion. Then $K$ is a finite direct product of field extensions of $F$. In this section, we consider a notion of $i_{K / F}: H_{q}^{*} F \rightarrow H_{q}^{*} K$ being not too far from an isomorphism. This notion depends on a positive integer $n$.

Definition 3.1. Let $K$ be a commutative semisimple $F$-algebra. We say that $K$ is $n$-taut over $F$ if both of the following conditions hold:

(i) $i_{K / F}: H_{q}^{n} F \rightarrow H_{q}^{n} K$ is surjective.

(ii) $i_{K / F}: H_{q}^{n+1} F \rightarrow H_{q}^{n+1} K$ is injective. 
Example 3.2. Let $F$ be a number field with exactly $r$ real completions $K_{1}, \ldots$, $K_{r}$. Let $K$ be the direct product of $K_{1}, \ldots, K_{r}$. Then $K$ is 2-taut over $F$ (cf. [T, §3.1]).

In the special case where $K$ equals $F_{q}$ (or the product of finitely many copies of $F_{q}$ ), we see that $K$ being $n$-taut over $F$ means that $H_{q}^{n+1} F=0$, i.e., that the cohomological 2-dimension of $\operatorname{Gal}\left(F_{q} / F\right)$, denoted by $\mathrm{cd}_{2}\left(\mathrm{Gal}\left(F_{q} / F\right)\right)$, is at most $n$. That, in turn, implies that $H_{q}^{m} E=0$ for every finite 2-extension $E$ of $F$ and every $m>n$. Our first goal in this section is to establish a corresponding result in the general case.

We begin with a 'Going up' result.

Proposition 3.3. Let $K$ be an $n$-taut commutative semisimple $F$-algebra. Let $E / F$ be a finite 2-extension and let $L=E \otimes_{F} K$. Then $L$ is $n$-taut over $E$. Proof. By induction on $[E: F]$, we reduce to the case when $[E: F]=2$. The result now follows by chasing in the commutative exact diagram:

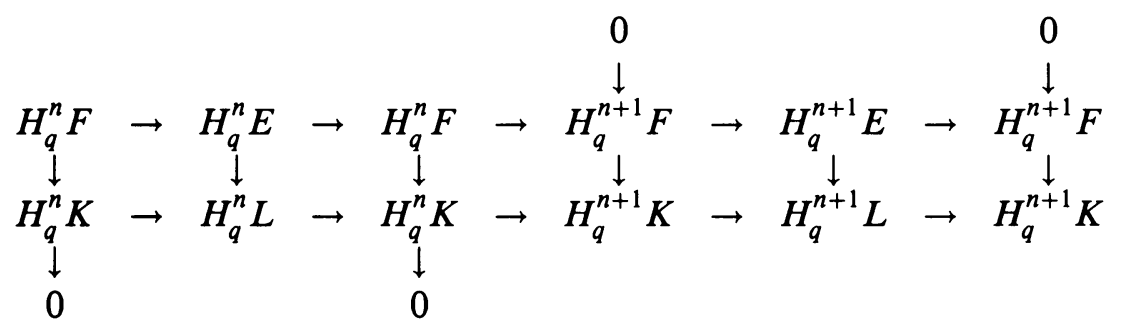

A similar proof now yields the following

Corollary 3.4. Let $K$ be an $n$-taut commutative semisimple $F$-algebra. Let $E / F$ be a finite 2-extension and let $L=E \otimes_{F} K$. Then the map from $\operatorname{ker}\left(H_{q}^{n} E \rightarrow H_{q}^{n} L\right)$ to $\operatorname{ker}\left(H_{q}^{n} F \rightarrow H_{q}^{n} K\right)$ induced by $\operatorname{cor}_{E / F}$ is surjective and the map from coker $\left(H_{q}^{n+1} F \rightarrow H_{q}^{n+1} K\right)$ to $\operatorname{coker}\left(H_{q}^{n+1} E \rightarrow H_{q}^{n+1} L\right)$ induced by $i_{E / F}$ is injective.

For our next proposition, we need two lemmas.

Lemma 3.5. Let $K$ be an $n$-taut commutative semisimple $F$-algebra. Let $K_{i}$ be one of the factors in the decomposition of $K$ into a direct product of fields. Let $M$ be a field composite of $F_{q}$ and $K_{i}$. Then $H_{q}^{n} M=0$.

Proof. Let $E / F$ be a finite 2-extension contained in $F_{q}$ and let $L=E \otimes_{F} K$. Let $L_{i}$ be the field composite of $E$ and $K_{i}$ in $M$. Then $L_{i}$ is one of the factors of $L$ in its decomposition into a direct product of fields. Let $H_{q}^{n} L \rightarrow H_{q}^{n} L_{i}$ be the projection map. Then the diagram

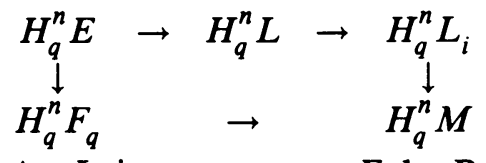

is clearly commutative. As $L$ is $n$-taut over $E$ by Proposition 3.3, the maps along the top row are surjective. Since $H_{q}^{n} F_{q}=0$, it follows that $H_{q}^{n} L_{i} \rightarrow H_{q}^{n} M$ 
is the zero map. As $H_{q}^{n} M$ is the direct limit of these $H_{q}^{n} L_{i}$, it follows that $H_{q}^{n} M=0$.

Lemma 3.6. Let $K$ be an $n$-taut commutative semisimple $F$-algebra. Let $\gamma \in$ $H_{q}^{n+1} K$. Then there exists a finite 2-extension $E / F$ such that $i_{E \otimes_{F} K / K}(\gamma)=0$. Proof. Let $K=\prod_{i=0}^{m} K_{i}$ be the decomposition of $K$ into a direct product of fields. Fix an $i$ and let $\gamma_{i}$ be the image of $\gamma$ in $H_{q}^{n+1} K_{i}$. Let $M$ be a field composite of $F_{q}$ and $K_{i}$. By the last lemma, $H_{q}^{n} M=0$, hence, by [S, Proposition I.11], also $H_{q}^{n+1} M=0$. It follows that there exists a finite 2extension $E_{i} / F$ such that if $L_{i}$ denotes the field composite of $E_{i}$ and $K_{i}$ in $M$ then $i_{L_{i} / K_{i}}\left(\gamma_{i}\right)=0$. Enlarging $E_{i}$, if necessary, we may assume that $E_{i} / F$ is Galois. Then all field composites of $E_{i}$ and $K_{i}$ are isomorphic over $K_{i}$, so $i_{L / K_{i}}\left(\gamma_{i}\right)=0$ for any field composite $L$ of $E_{i}$ and $K_{i}$. Let $E / F$ be a finite Galois 2-extension containing $E_{i}$ for all $i$. Then this $E$ works.

We can now proceed to prove the following

Proposition 3.7. Let $K$ be an $n$-taut commutative semisimple $F$-algebra. Then $K$ is $m$-taut over $F$ for all $m \geq n$.

Proof. By induction on $m$, it suffices to show that $K$ is $(n+1)$-taut over $F$. We first show that $i_{K / F}: H_{q}^{n+1} F \rightarrow H_{q}^{n+1} K$ is surjective. Let $\gamma \in H_{q}^{n+1} K$. By Lemma 3.6, there exists a finite 2-extension $E$ of $F$ such that $i_{L / K}(\gamma)=0$ where $L=E \otimes_{F} K$. In particular, $i_{L / K}(\gamma)$ lies in the image of $i_{L / E}: H_{q}^{n+1} E \rightarrow$ $H_{q}^{n+1} L$. By Corollary 3.4, it follows that $\gamma$ lies in the image of $i_{K / F}: H_{q}^{n+1} F \rightarrow$ $H_{q}^{n+1} K$ as needed.

We next show that $i_{K / F}: H_{q}^{n+2} F \rightarrow H_{q}^{n+2} K$ is injective. Let $\gamma \in H_{q}^{n+2} F$ satisfy $i_{K / F}(\gamma)=0$. There exists a finite 2-extension $E$ of $F$ such that $i_{E / F}(\gamma)=0$. By induction on $[E: F]$ and Proposition 3.3, we may assume that $E=F(\sqrt{d})$ is a quadratic extension of $F$. Let $L=E \otimes_{F} K$. Then the commutative diagram

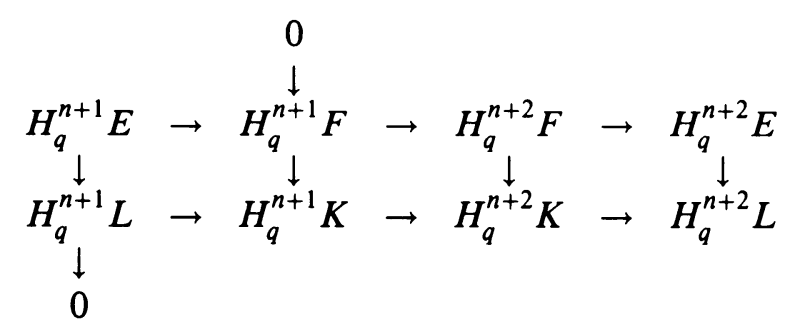

has exact rows. The columns are also exact. Indeed, the first column is exact by Proposition 3.3 and the first part of the proof while the second column is exact by hypothesis. The conclusion that $\gamma=0$ now follows by chasing in this diagram. 
It should be noted that, in contrast to the proposition, $H_{q}^{n} F \rightarrow H_{q}^{n} K$ an isomorphism does not imply that $H_{q}^{m} F \rightarrow H_{q}^{m} K$ is an isomorphism for all $m \geq n$.

Example 3.8. Let $F$ be a euclidean closure of $\mathbf{Q}$. Then $H_{q}^{2} F \neq 0$. Let $M$ be an odd degree extension field of $F$ that is not pythagorean (e.g., let $M=$ $F(\sqrt[3]{2})$ ). Then there exists a quadratic extension $M^{\prime}$ of $M$ that is not formally real but does not contain $\sqrt{-1}$. Let $K$ be a maximal algebraic extension of $M^{\prime}$ such that -1 is not a square in $K$. Then $H_{q}^{1} F \rightarrow H_{q}^{1} K$ is an isomorphism but $H_{q}^{2} K=0$. To generalize this example let

$$
\hat{F}=F\left(\left(t_{1}\right)\right) \cdots\left(\left(t_{n-1}\right)\right)
$$

and

$$
\hat{K}=K\left(\left(t_{1}\right)\right) \cdots\left(\left(t_{n-1}\right)\right) .
$$

Then $H_{q}^{n} \hat{F} \rightarrow H_{q}^{n} \hat{K}$ is an isomorphism but $H_{q}^{n-1} \hat{K}=0$ although $H_{q}^{n+1} \hat{F} \neq 0$.

Proposition 3.3 and Proposition 3.7 together now yield the desired result:

Theorem 3.9. Let $K$ be an $n$-taut commutative semisimple $F$-algebra. Let $E$ be a finite 2-extension of $F$ and let $L=E \otimes_{F} K$. Then $i_{L / E}: H_{q}^{m} E \rightarrow H_{q}^{m} L$ is surjective for $m=n$ and an isomorphism for all $m>n$.

As remarked earlier, $F_{q}$ being $n$-taut over $F$ simply means that

$$
\operatorname{cd}_{2}\left(\operatorname{Gal}\left(F_{q} / F\right)\right) \leq n \text {. }
$$

Hence Theorem 3.9 might be interpreted as a result about some notion of a relative cohomological dimension between $\operatorname{Gal}\left(F_{q} / F\right)$ and $\operatorname{Gal}\left(K_{q} / K\right)$. Indeed there is a purely group theoretic definition of such a relative cohomological dimension and a group theoretical proof of a theorem giving Theorem 3.9 (cf. [A4]).

In the 'absolute' case when $K$ equals $F_{q}$ (or the product of finitely many copies of $F_{q}$ ), Proposition 3.3 says that

$$
\operatorname{cd}_{2}\left(\operatorname{Gal}\left(E_{q} / E\right)\right) \leq \operatorname{cd}_{2}\left(\operatorname{Gal}\left(F_{q} / F\right)\right) .
$$

In this case, there is also a 'Going down' result giving the opposite inequality, provided $F$ is not formally real. We now look into the possibility of an analogous 'Going down' result in our 'relative' setting. For the basic case of a quadratic extension, we have the following technical result:

Lemma 3.10. Let $K$ be a commutative semisimple $F$-algebra. Let $E=F(\sqrt{d})$ be a quadratic extension of $F$ and let $L=E \otimes_{F} K$. Assume that $L$ is $n$-taut over $E$. Denote by $A^{m}$ the kernel of $i_{K / F}: H_{q}^{m} F \rightarrow H_{q}^{m} K$ and by $C^{m}$ the cokernel. Then for all $m \geq n$ we have

(1) (d) $\cup: A^{m} \rightarrow A^{m+1}$ is surjective.

(2) (d) $\cup: C^{m} \rightarrow C^{m+1}$ is injective.

(3) $\operatorname{ker}\left((d) \cup: A^{m+1} \rightarrow A^{m+2}\right)$ is isomorphic to $\operatorname{coker}\left((d) \cup: C^{m} \rightarrow C^{m+1}\right)$. 
Proof. Using Proposition 2.1, Proposition 2.2, and Proposition 3.7, we see that the following commutative diagram is exact:

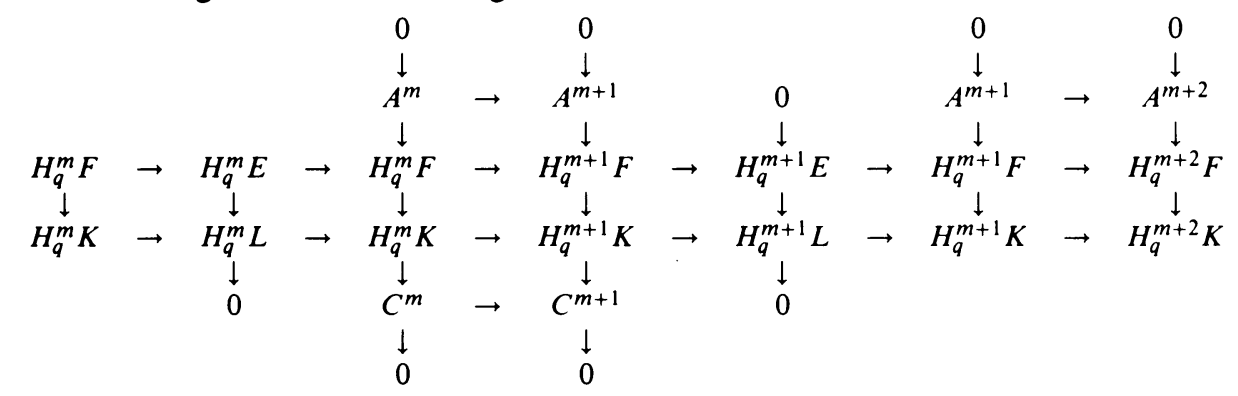

The results now follow by diagram chasing. (We do not use the left most square.)

A finite algebraic extension $E$ of $F$ is said to be totally real (over $F$ ) if each ordering of $F$ has $[E: F]$ extensions to orderings of $E$. In particular, a quadratic extension $E=F(\sqrt{d})$ of $F$ is totally real if and only if $d$ is totally positive in $F$, i.e., $d$ is a sum of squares in $F$. Furthermore, a finite 2-extension $E$ of $F$ is totally real if and only if $E$ is obtained from $F$ by successive totally real quadratic extensions. If $F$ is not formally real then, by this definition, every finite algebraic extension of $F$ is totally real. Hence the following 'Going down' result is a generalization of the 'Going down' result for cohomological dimension mentioned above.

Proposition 3.11. Let $K$ be a commutative semisimple $F$-algebra. Let $E$ be a totally real finite 2-extension of $F$ and let $L=E \otimes_{F} K$. Assume that $L$ is $n$-taut over $E$. Then $K$ is $n$-taut over $F$.

Proof. By induction on $[E: F]$, we may assume that $E=F(\sqrt{d})$ is a quadratic extension of $F$ where $d$ is a sum of squares in $F$. Then there is a $k$ such that $(-1)^{k} \cup(d)=0$. Using the well-known identity $(d) \cup(d)=(-1) \cup(d)$, we see that $(d)^{k+1}=0$. We now use Lemma 3.10 and the notation therein. By part (2) of this lemma, $(d)^{k+1} \cup: C^{m} \rightarrow C^{m+k+1}$ is injective. Thus $C^{m}=0$ for all $m \geq n$. Applying part (3) of this same lemma, it now follows that $(d) \cup: A^{m+1} \rightarrow A^{m+2}$ is injective. Using again that $(d)^{k+1}=0$, it now follows that $A^{m+1}=0$. This means that $K$ is $m$-taut over $F$ for all $m \geq n$.

Remark 3.12. The last proposition is false if we do not assume the extension is totally real. For example, let $F=\mathbf{R}(t)$ and $K=\mathbf{R}\left(t, \sqrt{-\left(1+t^{2}\right)}\right)$. Then $H_{q}^{n} F \neq 0$ but $H_{q}^{n} K=0$ for all $n \geq 2$. Thus $K$ cannot be $n$-taut over $F$ for any $n$. However, $K(\sqrt{-1})$ is 2-taut over $F(\sqrt{-1})$.

If $F$ is formally real then $H_{q}^{m} F \neq 0$ for all $m$. But we may have

$$
H_{q}^{n+1} F(\sqrt{-1})=0 \text {. }
$$

In this case we get, however, from the exact sequence $(\Delta)$ that $H_{q}^{n+1} F=(-1) \cup$ $H_{q}^{n} F$ and that $(-1) \cup: H_{q}^{n+1} F \rightarrow H_{q}^{n+2} F$ is injective. There are several ways to 
define something analogous in our 'relative' setting. Here we choose a definition that is suitable for the purposes of this paper.

To simplify the notation, we let $H_{q}^{n}(K / F):=\operatorname{ker}\left(i_{K / F}: H_{q}^{n} F \rightarrow H_{q}^{n} K\right)$ for any commutative semisimple $F$-algebra $K$.

Definition 3.13. Let $K$ be a commutative semisimple $F$-algebra. We say that $K$ is loosely $n$-taut over $F$ if all three of the following conditions hold:

(i) $i_{K / F}: H_{q}^{n} F \rightarrow H_{q}^{n} K$ is surjective.

(ii) $H_{q}^{n+1}(K / F)=(-1) \cup H_{q}^{n}(K / F)$.

(iii) $(-1) \cup: H_{q}^{n+1}(K / F) \rightarrow H_{q}^{n+2}(K / F)$ is injective.

Example 3.14. Let $F_{0}$ be a formally real field such that $H_{q}^{n+1} F_{0}(\sqrt{-1})=0$. Let $F$ be the pythagorean closure of $F_{0}$ (i.e., the smallest field extension of $F$ in which every sum of squares is a square). Then $H_{q}^{n+1} F(\sqrt{-1})=0$. Let $K$ be a SAP (cf. [EL2, Definition 1.4]) pythagorean field extending $F$ such that the inclusion map $F_{0} \subseteq K$ induces a bijection, hence a homeomorphism, between the spaces of orderings of $K$ and of $F_{0}$. (Such a $K$ exists by [Cr, Theorem].) Using that $I^{2} K(\sqrt{-1})=0$ by [EL2, Theorem 5.3 and EP, Theorem 3.3], it is easy to see that $K$ is loosely $n$-taut over $F$. But, in general, $K$ is not $n$-taut over $F$. Using also [A2, Satz 2], it can be seen that $K$ is $n$-taut over $F_{0}$.

Let $K$ be a commutative semisimple $F$-algebra. Clearly, if $K$ is $n$-taut over $F$ then $K$ is loosely $n$-taut over $F$. If -1 is a square in $F$ the converse also holds because then $(-1) \cup$ is the zero map. As we shall see this remains true whenever $F$ is not formally real. (Cf. Corollary 3.25 below.)

It is, of course, natural that the square class of -1 plays a special role in studying $H_{q}^{*} F$. It can, however, be shown that a commutative semisimple $F$ algebra $K$ is loosely $n$-taut over $F$ if and only if there exists an element $d$ in $\dot{F}$ satisfying the following three conditions:

(1) $i_{K / F}: H_{q}^{n} F \rightarrow H_{q}^{n} K$ is surjective.

(2) $H_{q}^{n+1}(K / F)=(d) \cup H_{q}^{n}(K / F)$.

(3) $(d) \cup: H_{q}^{n+1}(K / F) \rightarrow H_{q}^{n+2}(K / F)$ is injective.

The proof is an easy application of the identity $(d) \cup(d)=(-1) \cup(d)$. (By the same method, it can be shown that (3) just above can be replaced by (iii) in the definition of loosely $n$-taut.)

We shall not need this alternate characterization of loosely $n$-taut. We shall, however, use the following trivial observation.

Remark 3.15. Let $K$ be a commutative semisimple $F$-algebra. Then $K$ is loosely $m$-taut over $F$ for all $m \geq n$ if and only if $i_{K / F}: H_{q}^{m} F \rightarrow H_{q}^{m} K$ is surjective for all $m \geq n$ and $(-1) \cup: H_{q}^{m}(K / F) \rightarrow H_{q}^{m+1}(K / F)$ is surjective for $m=n$ and is an isomorphism for all $m>n$. 
We now want to prove a result for loose tautness corresponding to Theorem 3.9 for tautness. We start with a special case of 'Going up'.

Lemma 3.16. Let $K$ be a loosely $n$-taut commutative semisimple $F$-algebra. Let $F^{+}=F(\sqrt{-1})$ and let $K^{+}=F^{+} \otimes_{F} K$. Then $K^{+}$is $n$-taut over $F^{+}$.

Proof. As remarked above, $\sqrt{-1} \in F$ implies that $K$ is $n$-taut over $F$. Hence, we may assume that $\sqrt{-1} \notin F$. As $K$ is loosely $n$-taut over $F$, the following commutative diagram is exact:

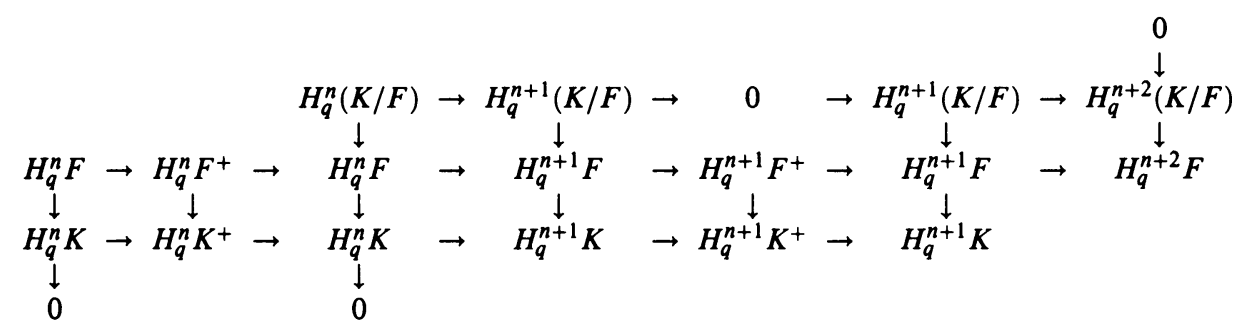

Chasing in this diagram, we see that $K^{+}$is $n$-taut over $F^{+}$.

We also have a 'Going down' result that includes a partial converse to the previous lemma.

Proposition 3.17. Let $K$ be a commutative semisimple $F$-algebra such that $i_{K / F}: H_{q}^{n} F \rightarrow H_{q}^{n} K$ is surjective. Let $F^{+}=F(\sqrt{-1})$ and let $K^{+}=F^{+} \otimes_{F} K$. If $K^{+}$is $n$-taut over $F^{+}$then $K$ is loosely $m$-taut over $F$ for all $m \geq n$.

Proof. We first show that $i_{K / F}: H_{q}^{m} F \rightarrow H^{m} K$ is surjective. Let $\lambda \in H_{q}^{m} K$. By [A2 or AEJ1, Theorem 5.13 (iii)], there is a $k$ such that $(-1)^{k} \cup \lambda$ lies in the subring of $H_{q}^{*} K$ generated by $H_{q}^{1} K$. Enlarging $k$, we may assume that $m+k$ is divisible by $n$. Then $(-1)^{k} \cup \lambda$ lies in the subring of $H_{q}^{*} K$ generated by $H_{q}^{n} K$. As $i_{K / F}: H_{q}^{n} F \rightarrow H_{q}^{n} K$ is surjective, it follows that $(-1)^{k} \cup \lambda$ lies in the image of $i_{K / F}: H_{q}^{m+k} F \rightarrow H_{q}^{m+k} K$. By part (2) of Lemma 3.10, cup product with $(-1)^{k}$ from $\operatorname{coker}\left(i_{K / F}: H_{q}^{m} F \rightarrow H_{q}^{m} K\right)$ to $\operatorname{coker}\left(i_{K / F}: H_{q}^{m+k} F \rightarrow H_{q}^{m+k} K\right)$ is injective. Thus $\lambda$ lies in the image of $i_{K / F}: H_{q}^{m} F \rightarrow H_{q}^{m} K$ as needed.

Applying part (3) of Lemma 3.10, it now follows that the map

$$
(-1) \cup: H_{q}^{m+1}(K / F) \rightarrow H_{q}^{m+2}(K / F)
$$

is injective. By part (1) of Lemma 3.10,

$$
(-1) \cup: H_{q}^{m}(K / F) \rightarrow H_{q}^{m+1}(K / F)
$$

is surjective.

The following corollary is really a stronger version of the proposition. We shall, however, not need it in the sequel. 
Corollary 3.18. Let $K$ be a commutative semisimple $F$-algebra. Let $E$ be a finite 2-extension of $F$ and let $L=E \otimes_{F} K$. If $L$ is loosely $n$-taut over $E$ and $i_{K / F}: H_{q}^{n} F \rightarrow H_{q}^{n} K$ is surjective then $K$ is loosely $m$-taut over $F$ for all $m \geq n$.

Proof. Let $F^{+}=F(\sqrt{-1})$ and let $K^{+}=F^{+} \otimes_{F} K$. Define $E^{+}$and $L^{+}$in a similar way. By Lemma 3.16, $L^{+}$is $n$-taut over $E^{+}$and hence by Proposition $3.11, K^{+}$is $n$-taut over $F^{+}$. The result now follows by the proposition above.

Remark 3.19. In the proof of Proposition 3.17, we did not need the full force of the hypothesis that $i_{K / F}: H_{q}^{n} F \rightarrow H_{q}^{n} K$ is surjective. We only used that the subgroup of $H_{q}^{n} K$ generated by $n$-fold cup products of elements in $H_{q}^{1} K$ lies in the image of $i_{K / F}: H_{q}^{n} F \rightarrow H_{q}^{n} K$. We shall need this stronger formulation later on.

Lemma 3.16 and the last proposition immediately imply the following analog of Proposition 3.7.

Proposition 3.20. Let $K$ be a loosely $n$-taut commutative semisimple $F$-algebra. Then $K$ is loosely $m$-taut over $F$ for all $m \geq n$.

We can now prove our general 'Going up' result.

Proposition 3.21. Let $K$ be a loosely $n$-taut commutative semisimple $F$-algebra. Let $E$ be a finite 2-extension of $F$ and let $L=E \otimes_{F} K$. Then $L$ is loosely $n$-taut over $E$.

Proof. By induction on $[E: F]$, we may assume that $E=F(\sqrt{d})$ is a quadratic extension of $F$.

We first show that $i_{L / E}: H_{q}^{n} E \rightarrow H_{q}^{n} L$ is surjective. Let $\delta \in H_{q}^{n} L$. Chasing in the exact commutative diagram

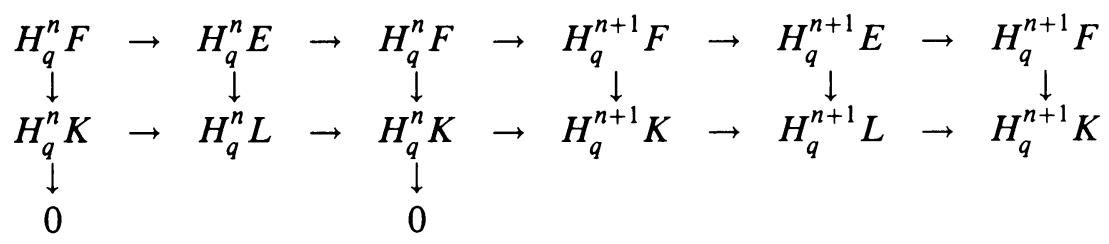

shows that $\operatorname{cor}_{L / K}(\delta)=i_{K / F}(\mu)$ for some $\mu \in H_{q}^{n} F$ and that, moreover, $(d) \cup \mu$ lies in $H_{q}^{n+1}(K / F)$. By condition (ii) in the definition of loosely $n$-taut, we then have $(d) \cup \mu=(-1) \cup \phi$ for some $\phi \in H_{q}^{n}(K / F)$. It follows that $0=$ $(-d) \cup(d) \cup \mu=(-d) \cup(-1) \cup \phi$. Hence, by condition (iii) in the definition of loosely $n$-taut, $(-d) \cup \phi=0$. Thus $(d) \cup \phi=(-1) \cup \phi=(d) \cup \mu$. Since $i_{K / F}(\phi)=0$, this argument shows that, replacing $\mu$ by $\mu-\phi$, we may assume that $(d) \cup \mu=0$. A straightforward chase in the diagram above now shows that $\delta$ lies in the image of $i_{L / E}: H_{q}^{n} E \rightarrow H_{q}^{n} L$. 
Now let $F^{+}=F(\sqrt{-1})$ and let $K^{+}=F^{+} \otimes_{F} K$. Define $E^{+}$and $L^{+}$in a similar way. By Lemma 3.16, $K^{+}$is $n$-taut over $F^{+}$and hence, by Proposition 3.3, $L^{+}$is $n$-taut over $E^{+}$. The result now follows by Proposition 3.17.

Corollary 3.22. Let $K$ be a loosely $n$-taut commutative semisimple $F$-algebra. Let $E=F(\sqrt{d})$ be a quadratic extension of $F$ and let $L=E \otimes_{F} K$. Then the sequence

$$
\begin{aligned}
H_{q}^{n}(L / E) & \rightarrow H_{q}^{n}(K / F) \rightarrow H_{q}^{n+1}(K / F) \rightarrow H_{q}^{n+1}(L / E) \\
& \rightarrow H_{q}^{n+1}(K / F) \rightarrow H_{q}^{n+2}(K / F) \rightarrow \ldots
\end{aligned}
$$

is exact.

Proof. Proposition 3.21 and a straightforward chase in the diagram above shows exactness at the first three places. The rest follows by using Proposition 3.20.

Our generalization of Theorem 3.9 now follows immediately from Proposition 3.20 and Proposition 3.21. To simplify the statement, we first make the following

Definition 3.23. A subgroup $H$ of $H_{q}^{n} F$ is said to be (-1)-torsion-free if there is no nonzero element $\alpha \in H$ such that $(-1)^{k} \cup \alpha=0$ for some $k$.

Theorem 3.24. Let $K$ be a loosely $n$-taut commutative semisimple $F$-algebra. Let $E$ be a finite 2-extension of $F$ and let $L=E \otimes_{F} K$. Then

(1) $i_{L / E}: H_{q}^{m} E \rightarrow H_{q}^{m} L$ is surjective for all $m \geq n$.

(2) $H_{q}^{m}(L / E)=(-1)^{m-n} \cup H_{q}^{n}(L / E)$ for all $m>n$.

(3) $H_{q}^{m}(L / E)$ is $(-1)$-torsion-free for all $m>n$.

If $F$ is not formally real then $(-1)^{k}=0$ for some $k$. Hence we have the following

Corollary 3.25. Assume that $F$ is not formally real. Let $K$ be a commutative semisimple $F$-algebra. Then $K$ is loosely $n$-taut over $F$ if and only if $K$ is $n$-taut over $F$.

Combining the theorem with Proposition 3.17, we get the following specific example of a 'relative result':

Corollary 3.26. Let $K$ be a commutative semisimple $F$-algebra such that $i_{K / F}$ : $H_{q}^{n} F \rightarrow H_{q}^{n} K$ is surjective. Let $F^{+}=F(\sqrt{-1})$ and $K^{+}=F(\sqrt{-1}) \otimes_{F} K$. Then the following are equivalent:

(1) $H_{q}^{n+1}(K / F)=(-1) \cup H_{q}^{n}(K / F)$ and is (-1)-torsion-free.

(2) $i_{K^{+} / F^{+}}: H_{q}^{n} F^{+} \rightarrow H_{q}^{n} K^{+}$is surjective and $H_{q}^{n+1}\left(K^{+} / F^{+}\right)=0$.

\section{Tight Algebras}

In this section, we begin the study of the quadratic form theoretic analog of tautness. 
Definition 4.1. Let $K$ be a commutative semisimple $F$-algebra. We say that $K$ is $n$-tight over $F$ if the following two conditions hold:

(a) $i_{K / F}: I^{n} F \rightarrow I^{n} K$ is surjective.

(b) $i_{K / F}: I^{n+1} F \rightarrow I^{n+1} K$ is injective.

Examples 4.2. (1) Let $K=F_{q}$. Then $K$ is $n$-tight over $F$ if and only if $I^{n+1} F=0$.

(2) Let $F$ be a number field with exactly $r$ real completions $K_{1}, \ldots, K_{r}$. Let $K$ be the direct product of $K_{1}, \ldots, K_{r}$. Then $K$ is 2-tight over $F$ (cf. [Mi, Appendix or EL1, Example 4.3(2)]).

For convenience of notation, we let $I^{n}(K / F)=\operatorname{ker}\left(i_{K / F}: I^{n} F \rightarrow I^{n} K\right)$.

Definition 4.3. Let $K$ be a commutative semisimple $F$-algebra. We say that $K$ is loosely $n$-tight over $F$ if the following three conditions hold:

(1) $i_{K / F}: I^{n} F \rightarrow I^{n} K$ is surjective.

(2) $I^{n+1}(K / F)=2 I^{n}(K / F):=\langle 1,1\rangle \otimes I^{n}(K / F)$.

(3) $I^{n+1}(K / F)$ is torsion-free.

Examples 4.4. (1) Let $K=F_{q}$. Then $K$ is loosely $n$-tight over $F$ if and only if $I^{n+1} F=2 I^{n} F$ and $I^{n+1} F$ is torsion-free. If $F$ is formally real then $K$ is not $n$-tight over $F$.

(2) Let $F_{0}$ be a formally real field such that $I^{n+1} F_{0}=2 I^{n} F_{0}$. Let $F$ be the pythagorean closure of $F_{0}$. Using [EP, Theorem 3.1], one can show that $I^{n+1} F=2 I^{n} F$. Let $K$ be a pythagorean field extending $F$ such that the inclusion map $F_{0} \subseteq K$ induces a bijection, hence a homeomorphism, between the spaces of orderings of $K$ and of $F_{0}$. (Such a $K$ exists by [Cr, Theorem].) Then $K$ is loosely $n$-tight over $F$, but, in general, $K$ is not $n$-tight over $F$. If $I^{n+1} F_{0}$ is also torsion-free then $K$ is $n$-tight over $F_{0}$.

Let $K$ be a commutative semisimple $F$-algebra. Certainly, if $K$ is $n$-tight over $F$ then it is loosely $n$-tight over $F$. Conversely, if $F$ is not formally real and condition (3) above holds then condition (b) holds. Thus if $F$ is not formally real then $K$ loosely $n$-tight over $F$ implies that $K$ is $n$-tight over $F$. It can be useful to think of the $n$-tight condition as the 'relative nonreal' case, even though this condition can hold when $F$ itself is formally real.

We would like to prove results about tightness analogous to the ones proved about tautness in the preceding section. We are, however, not able to do this without some extra conditions.

Let $K$ be a commutative semisimple $F$-algebra. If $i_{K / F}: I^{n} F \rightarrow I^{n} K$ is surjective then $i_{K / F}: I^{m} F \rightarrow I^{m} K$ is surjective for every $m \geq n$. (Indeed by induction, we may assume that $m=n+1$. Then we have $I^{n+1} K=I K \otimes$ $\left.i_{K / F}\left(I^{n} F\right)=I K \otimes i_{K / F}\left(I^{n-1} F\right) \otimes i_{K / F}(I F) \subseteq I^{n} K \otimes i_{K / F}(I F)=i_{K / F}\left(I^{n+1} F\right).\right)$ 
It follows that if $K$ is $n$-tight over $F$ then $K$ is $m$-tight over $F$ for every $m \geq n$. But we have not been able to prove that $K$ loosely $n$-tight over $F$ implies that $K$ is also loosely $m$-tight over $F$ for all $m \geq n$. Note that this would mean that $I^{n+k}(K / F)=2^{k} I^{n}(K / F)$ for all $k \geq 1$.

Let $K$ be a commutative semisimple $F$-algebra. Let $E$ be a finite 2-extension of $F$ and let $L=E \otimes_{F} K$. We have not been able to prove that $K n$-tight over $F$ implies that $L$ is $n$-tight over $E$. (We have the same problem with loosely $n$-tight.)

The main reason for us not being able to get these desired results is that we do not know if the sequence $(\nabla)$ is always exact at the right places. Consequently, we shall need some substitute.

We would also like to prove that (loosely) $n$-tight over $F$ implies (loosely) $n$-taut over $F$. We are not able to do this without extra conditions. To explain this problem, let us look at the special case that $K=F_{q}$. Then $K$ being $n$-tight over $F$ simply means that $I^{n+1} F=0$ and $K$ being $n$-taut over $F$ means that $H_{q}^{n+1} F=0$. It is not known, however, if $I^{n+1} F=0$ implies that $H_{q}^{n+1} F=0$ (cf. Corollary 1.4).

In our applications, we shall assume that $\bar{e}_{q, F}^{m}$ is a well-defined isomorphism for all $m<n$ and that $e_{q, F}^{n}$ is well-defined for all fields $F$. (Cf. Corollary 1.4 and Proposition 2.6.) However, we do not need the full force of this assumption for the results of this section and the next one. We shall, therefore, work with weaker (but not as natural) hypotheses, which we now give a name.

Definition 4.5. Let $K$ be a commutative semisimple $F$-algebra. We say that $K$ is $n$-admissible over $F$ if the following two conditions hold:

(1) For every finite 2-extension $M$ of any field in the decomposition of $K$ into a direct product of fields, $e_{q, M}^{n}$ is well-defined and its image $\operatorname{im}\left(e_{q, M}^{n}\right)$ contains $H_{q}^{1} M \cup H_{q}^{n-1} M$.

(2) If $E / F$ is a finite 2-extension and $E^{\prime} / E$ is a quadratic extension then

$$
I^{n} L \rightarrow I^{n} L^{\prime} \rightarrow I^{n} L \rightarrow J_{n+1} L \rightarrow J_{n+1} L^{\prime} \rightarrow J_{n+1} L
$$

is exact where $L=E \otimes_{F} K$ and $L^{\prime}=E^{\prime} \otimes_{F} K$.

If $F$ is $n$-admissible over $F$, we simply say that $F$ is $n$-admissible.

Of course, condition (1) in the above definition guarantees that the same holds for arbitrary 2-extensions $M$.

Let $K$ be a commutative semisimple $F$-algebra. Let $E$ be a finite 2-extension of $F$ and let $L=E \otimes_{F} K$. It is then clear from the definition that $K$ being $n$-admissible over $F$ implies that $L$ is $n$-admissible over $E$. But we do not know, of course, if $K$ being $n$-admissible over $F$ implies that $K$ is $m$-admissible over $F$ for all $m \geq n$.

Examples 4.6. (1) Let $K$ be a commutative semisimple $F$-algebra. Then $K$ is easily seen to be 1 -admissible and 2-admissible over $F$. 
(2) Let $F=\mathbf{R}$ and $K$ be a field of transcendence degree at most five over $F$. Then $K(\sqrt{-1})$ is $n$-admissible over $F(\sqrt{-1})$ for all $n$ by Theorem 1.6 and Proposition 2.6. But at present it is not known if $K$ is 5 -admissible over $F$.

These examples are special cases of the following

Proposition 4.7. Let $K$ be a commutative semisimple $F$-algebra. Assume for any finite 2-extension $M$ of any field in the decomposition of $K$ into a direct product of fields that $\bar{e}_{q, M}^{m}$ is well-defined for all $m \leq n$ and is an isomorphism for all $m<n$. Then $K$ is $n$-admissible over $F$.

Proof. The first condition is immediate, since $e_{q, M}^{n-1}$ is surjective by hypothesis. The second condition follows from Proposition 2.6.

Corollary 4.8. Let $K$ be a commutative semisimple $F$-algebra. Then $K$ is $n$ admissible over $F$ for every $n \leq 4$.

Proof. This follows immediately from Proposition 4.7, using [A1 and Me] for $n=3$ and [MS or R and JR] for $n=4$.

For consistency of notation, we let $J_{n+1}(K / F)=\operatorname{ker}\left(i_{K / F}: J_{n+1} F \rightarrow J_{n+1} K\right)$ whenever both $e_{q, F}^{n}$ and $e_{q, K}^{n}$ are well-defined.

We can now formulate and prove our 'Going up' result.

Proposition 4.9. Let $K$ be a loosely $n$-tight commutative semisimple $F$-algebra. Assume that both $F$ and $K$ are $n$-admissible over $F$ and that $J_{n+1}(K / F)=$ $I^{n+1}(K / F)$. Let $E / F$ be a finite 2-extension and let $L=E \otimes_{F} K$. Then $L$ is loosely $n$-tight over $E$ and $J_{n+1}(L / E)=I^{n+1}(L / E)$.

Proof. By induction on $[E: F]$, we may assume that $E=F(\sqrt{d})$ is a quadratic extension of $F$. Consider the commutative diagram:

(D)

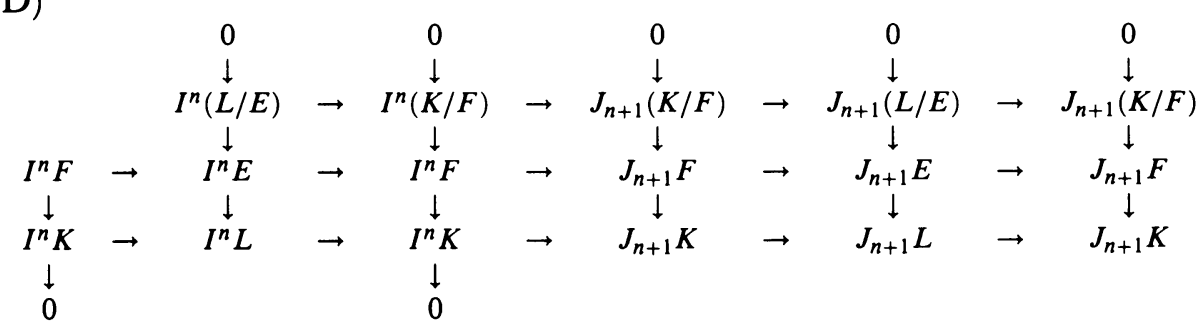

Since both $K$ and $F$ are $n$-admissible over $F$, the bottom two rows are exact. Since $K$ is loosely $n$-tight over $F$, the columns are exact.

We first show that $i_{L / E}: I^{n} E \rightarrow I^{n} L$ is surjective. Let $\phi \in I^{n} L$. By the exactness in diagram (D) there exists a $\sigma \in I^{n} F$ such that $i_{K / F}(\sigma)=s_{L / K}^{*}(\phi)$. Since $0=\langle 1,-d\rangle \otimes s_{L / K}^{*}(\phi)=i_{K / F}(\langle 1,-d\rangle \otimes \sigma)$, condition (2) in the definition of loosely $n$-tight produces a $\tau \in I^{n}(K / F)$ such that $2 \tau=\langle 1,-d\rangle \otimes \sigma$. Then $2\langle 1, d\rangle \otimes \tau=\langle 1,-d\rangle \otimes\langle 1, d\rangle \otimes \sigma=0$, so $\langle 1, d\rangle \otimes \tau$ is torsion. Hence 
$\langle 1, d\rangle \otimes \tau=0$ by condition (3) in the definition of loosely $n$-tight. Thus $\langle 1,-d\rangle \otimes \tau=2 \tau=\langle 1,-d\rangle \otimes \sigma$. This shows that, by replacing $\sigma$ by $\sigma-\tau$, we may assume that $\langle 1, d\rangle \otimes \sigma=0$. Chasing in diagram (D) now shows that $\phi$ lies in $i_{L / E}\left(I^{n} E\right)$ as needed.

We next show that $J_{n+1}(L / E) \subseteq 2 I^{n}(L / E)$ and hence that $J_{n+1}(L / E)=$ $I^{n+1}(L / E)=2 I^{n}(L / E)$. Let $\phi \in J_{n+1}(L / E)$. Since $s_{E / F}^{*}(\phi) \in J_{n+1}(K / F)$, by hypothesis there exists a $\sigma$ in $I^{n}(K / F)$ such that $s_{E / F}^{*}(\phi)=2 \sigma$. As $0=$ $\langle 1,-d\rangle \otimes s_{E / F}^{*}(\phi)=2\langle 1,-d\rangle \otimes \sigma$, the form $\langle 1,-d\rangle \otimes \sigma$ is torsion, hence by hypothesis zero. Chasing in diagram (D) shows that $\sigma=s_{E / F}^{*}(\tau)$ for some $\tau \in$ $I^{n}(L / E)$. This shows that, replacing $\phi$ by $\phi-2 \tau$, we may assume that $s_{E / F}^{*}(\phi)=0$. Further chasing in diagram (D) then shows that $\phi$ lies in $i_{E / F}\left(J_{n+1}(K / F)\right)=2 i_{E / F}\left(I^{n}(K / F)\right) \subseteq 2 I^{n}(E / F)$ as needed.

To finish, we must show that $I^{n+1}(L / E)$ is torsion-free. Since $I^{n+1}(L / E)=$ $2 I^{n}(L / E)$, it suffices to show that if $\phi \in I^{n}(L / E)$ satisfies $2^{m} \phi=0$ for some $m \geq 1$ then $2 \phi=0$. By hypothesis $2 s_{E / F}^{*}(\phi)=0$. Chasing in diagram (D) shows that $2 \phi=i_{E / F}(\sigma)$ for some $\sigma$ in $J_{n+1}(K / F)=2 I^{n}(K / F)$. Write $\sigma=$ $2 \mu$ for some $\mu$ in $I^{n}(K / F)$, so that $2 \phi=2 i_{E / F}(\mu)$. Then $i_{E / F}(\mu)$ is a torsion form. It follows that the signature of $\mu$ with respect to $\alpha$, denoted by $\operatorname{sgn}_{\alpha}(\mu)$, is zero for every ordering $\alpha$ on $F$ that extends to $E$. If $\alpha$ is an ordering on $F$ that does not extend to $E$ then $d<_{\alpha} 0$. Thus $\operatorname{sgn}_{\alpha}(\langle 1, d\rangle \otimes \mu)=0$ for every ordering $\alpha$ on $F$. It follows by the Pfister Local-Global Principle [P, Satz 22] that the form $\langle 1, d\rangle \otimes \mu$ in $I^{n+1}(K / F)$ is torsion, hence by hypothesis zero. Consequently, $2 \mu=\langle 1,-d\rangle \otimes \mu$. This shows that $2 \phi=i_{E / F}((2 \mu))=0$ as needed.

Corollary 4.10. Let $K$ be a loosely $n$-tight commutative semisimple $F$-algebra. Assume that both $F$ and $K$ are $n$-admissible over $F$ and that $J_{n+1}(K / F)=$ $I^{n+1}(K / F)$. Let $E=F(\sqrt{d})$ be a quadratic extension of $F$ and let $L=E \otimes_{F} K$. Then the sequence

$$
I^{n}(L / E) \rightarrow I^{n}(K / F) \rightarrow I^{n+1}(K / F) \rightarrow I^{n+1}(L / E) \rightarrow I^{n+1}(K / F) \rightarrow I^{n+2}(K / F)
$$

is exact.

Proof. Using the surjectivity of $i_{K / F}: I^{n} F \rightarrow I^{n} K$ and $i_{L / E}: I^{n} E \rightarrow I^{n} L$, chasing in diagram (D) shows that its top row is exact. Since

$$
J_{n+1}(K / F)=I^{n+1}(K / F) \text { and } J_{n+1}(L / E)=I^{n+1}(L / E),
$$

there remains only to prove the exactness at the second occurrence of $I^{n+1}(K / F)$. Suppose that $\phi \in I^{n+1}(K / F)$ satisfies $\langle 1,-d\rangle \otimes \phi=0$. Write $\phi=2 \psi$ for some $\psi \in I^{n}(K / F)$. It follows that $\langle 1,-d\rangle \otimes \psi$ is torsion, hence zero. By the exactness shown so far, $\psi=s_{E / F}^{*}(\mu)$ for some $\mu \in I^{n}(L / E)$. It follows that $\phi=s_{E / F}^{*}(2 \mu)$ as needed. 
Corollary 4.11. Let $K$ be an $n$-tight commutative semisimple $F$-algebra. Assume that both $F$ and $K$ are $n$-admissible over $F$ and that $J_{n+1}(K / F)=$ $I^{n+1}(K / F)$. Let $E / F$ be a finite 2-extension and let $L=E \otimes_{F} K$. Then $L$ is $n$-tight over $E$ and $J_{n+1}(L / E)=I^{n+1}(L / E)$.

Proof. As before, we may assume that $E / F$ is a quadratic extension. By the proposition, $L$ is loosely $n$-tight over $E$. Since $I^{n+1}(K / F)=0$, we have $I^{n+1}(L / E)=0$ by Corollary 4.10 .

Corollary 4.12. Let $K$ be a loosely $m$-tight commutative semisimple $F$-algebra for all $m \geq n$. Assume that both $F$ and $K$ are $n$-admissible over $F$ and that $J_{n+1}(K / F)=I^{n+1}(K / F)$. Let $E / F$ be a finite 2-extension and let $L=E \otimes_{F} K$. Then $L$ is loosely $m$-tight over $E$ over all $m \geq n$ and $J_{n+1}(L / E)=I^{n+1}(L / E)$.

Proof. It suffices to show $I^{m}(L / E)=2^{m-n} I^{n}(L / E)$ for all $m>n$. It suffices to do the case when $E=F(\sqrt{d})$ is a quadratic extension of $F$. Let $\psi \in I^{m}(L / E)$. We can replace $\psi$ by any element in its coset modulo $2^{m-n} I^{n}(L / E)$. We know that $s_{E / F}^{*}(\psi) \in I^{m}(K / F)$ hence that $s_{E / F}^{*}(\psi)=2^{m-n} \mu$ for some $\mu \in I^{n}(K / F)$ by hypothesis. Since $0=\langle 1,-d\rangle \otimes s_{E / F}^{*}(\psi)=2^{m-n}\langle 1,-d\rangle \otimes \mu$, the form $\langle 1,-d\rangle \otimes \mu$ is torsion, hence zero. By Corollary 4.10 , we have $\mu=s_{E / F}^{*}(\rho)$ for some $\rho \in I^{n}(L / E)$. Replacing $\psi$ by $\psi-2^{m-n} \rho$, we see that, modulo $2^{m-n} I^{n}(L / E)$, we may assume that $s_{E / F}^{*}(\psi)=0$. Corollary 4.10 now implies that $\psi=i_{E / F}(2 \phi)$ for some $\phi \in I^{n}(K / F)$, using the assumption $I^{n+1}(K / F)=$ $2 I^{n}(K / F)$. Now $\psi \in I^{m} E$ implies that $2^{m-1} \mid \operatorname{sgn}_{\alpha}(\phi)$ for any ordering $\alpha$ of $F$ that extends to $E$. Since $\operatorname{sgn}_{\alpha}(\langle 1, d\rangle)=0$ if the ordering $\alpha$ of $F$ does not extend to $E$, we have $2^{m} \mid \operatorname{sgn}_{\alpha}(\langle 1, d\rangle \otimes \phi)$ for every ordering $\alpha$ of $F$. Thus, by [AEJ1, Proposition 2.1], there exists a $k$ such that $2^{k}\langle 1, d\rangle \otimes \phi \in I^{k+m}(K / F)=$ $2^{k+m-n} I^{n}(K / F)$. Since $I^{n+1}(K / F)$ is torsion-free, we see that $\langle 1, d\rangle \otimes \phi \in$ $2^{m-n} I^{n}(K / F)$. Hence $\psi=i_{E / F}(2 \phi)=i_{E / F}(\langle 1, d\rangle \otimes \phi) \in 2^{m-n} I^{n}(K / F)$ as needed.

Corollary 4.13. Let $n \leq 3$. Let $K$ be an $n$-tight (respectively, loosely $n$-tight) commutative semisimple $F$-algebra. Let $E / F$ be a finite 2-extension and let $L=E \otimes_{F} K$. Then $L$ is $n$-tight (respectively, loosely $n$-tight) over $E$.

Proof. This follows immediately from Proposition 4.9, since $K$ is $n$-admissible over $F$ by Corollary 4.8 and since $J_{n+1} F=I^{n+1} F$ as previously remarked.

\section{From tight to taUt}

This purpose of this section is to show that, under suitable additional hypotheses, a (loosely) $n$-tight $F$-algebra is also (loosely) $n$-taut over $F$. We begin by looking at the relevant kernels. 
Proposition 5.1. Let $K$ be a loosely $n$-tight commutative semisimple $F$-algebra. Assume that both $F$ and $K$ are $n$-admissible over $F$ and that $J_{n+1}(K / F)=$ $I^{n+1}(K / F)$. Then the map $I^{n}(K / F) \rightarrow H_{q}^{n}(K / F)$ induced by $e_{q, F}^{n}$ is surjective. Proof. Let $\chi \in H_{q}^{n}(K / F)$. There exists a finite 2-extension $E / F$ such that $i_{E / F}(\chi)=e_{q, E}^{n}(\phi)$ for some $\phi \in I^{n}(L / E)$ where $L=E \otimes_{F} K$. (For example, there exists such an $E$ with $i_{E / F}(\chi)=0$.) By Proposition 4.9 and induction on $[E: F]$, we may assume that $E=F(\sqrt{d})$ is a quadratic extension of $F$. Since

$$
e_{q, F}^{n}\left(s_{E / F}^{*}(\phi)\right)=\operatorname{cor}_{E / F}\left(e_{q, E}^{n}(\phi)\right)=\operatorname{cor}_{E / F}\left(i_{E / F}(\chi)\right)=0,
$$

the form $s_{E / F}^{*}(\phi)$ lies in $J_{n+1} F \cap I^{n}(K / F)=J_{n+1}(K / F)=I^{n+1}(K / F)$. Thus $s_{E / F}^{*}(\phi)=s_{E / F}^{*}(\gamma)$ for some $\gamma$ in $J_{n+1}(L / E)$ by Corollary 4.10. Hence, replacing $\phi$ by $\phi-\gamma$, we may assume that, in addition, $s_{E / F}^{*}(\phi)=0$. Since $F$ is $n$-admissible over $F$, we then have that $\phi=i_{E / F}(\mu)$ for some $\mu \in$ $I^{n} F$. It follows by the exactness of the sequence $(\Delta)$ that $\chi-e_{q, F}^{n}(\mu)$ lies in $(d) \cup H_{q}^{n-1} F$. Hence condition (1) in the definition of $n$-admissible over $F$ implies that we may write $\chi-e_{q, F}^{n}(\mu)=e_{q, F}^{n}(\rho)$ for some $\rho \in I^{n} F$. Moreover, $i_{E / F}(\rho) \in J_{n+1} E$ and $s_{E / F}^{*}\left(i_{E / F}(\rho)\right)=0$, so that the $n$-admissibility of $F$ implies that $i_{E / F}(\rho)$ lies in the image of $i_{E / F}: J_{n+1} F \rightarrow J_{n+1} E$. This shows that, by modifying $\rho$ by an element of $J_{n+1} F$, we may assume that $\chi-e_{q, F}^{n}(\mu)=e_{q, F}^{n}(\rho)$ and $i_{E / F}(\rho)=0$. Thus replacing $\mu$ by $\mu-\rho$, we see that we may assume that $\chi=e_{q, F}^{n}(\mu)$ and $i_{E / F}(\mu)=\phi$. We must still show that $\mu$ can be chosen such that $i_{K / F}(\mu)=0$. Certainly, $i_{K / F}(\mu)$ lies in $J_{n+1} K$ and $i_{L / F}(\mu)=i_{L / E}\left(i_{E / F}(\mu)\right)=i_{L / E}(\phi)=0$. Condition (2) in the definition of the $n$-admissibility of $K$ therefore shows that $i_{E / F}(\mu)$ lies in $\langle 1,-d\rangle \otimes I^{n} K$. Since $i_{K / F}: I^{n} F \rightarrow I^{n} K$ is surjective, $i_{E / F}(\mu)=\langle 1,-d\rangle \otimes i_{K / F}(\tau)$ for some $\tau \in I^{n} F$. Now $i_{E / F}(\mu-\langle 1,-d\rangle \otimes \tau)=\phi$ and $\langle 1,-d\rangle \otimes \tau \in I^{n+1} F \subseteq J_{n+1} F$. Thus $e_{q, F}^{n}(\mu-\langle 1,-d\rangle \otimes \tau)=\chi$, so we may indeed assume that $i_{E / F}(\mu)=0$ as needed.

We have the following analogs of Lemma 3.5 and Lemma 3.6:

Lemma 5.2. Let $K$ be a loosely $n$-tight commutative semisimple $F$-algebra. Assume that both $F$ and $K$ are $n$-admissible over $F$ and that $J_{n+1}(K / F)=$ $I^{n+1}(K / F)$. Let $K_{i}$ be one of the factors of $K$ in its decomposition into a direct product of fields. Let $M$ be a field composite of $F_{q}$ and $K_{i}$. Then $I^{n} M=0$ and $H_{q}^{n} M=0$.

Proof. By replacing $H_{q}^{n}$ in the proof of Lemma 3.5 by $I^{n}$, we see that $I^{n} M=$ 0 . Since $I^{n} M=0$, we have $I^{n} N=0$ for every 2-extension $N$ of $M$ by [A1, Satz 3.6 or EL3, Theorem 6.3]. Let $\alpha \in H_{q}^{n} M$. We must show that $\alpha=0$. There exists a finite 2-extension $N$ of $M$ such that $i_{N / M}(\alpha)=0$. By induction, 
we may assume that $N / M$ is a quadratic extension, say $N=M(\sqrt{d})$. But then we have $\alpha=(d) \cup \beta$ for some $\beta$ in $H_{q}^{n-1} M$. Condition (1) in the definition of $n$-admissibility for $K$ implies that $H_{q}^{1} M \cup H_{q}^{n-1} M \subseteq e_{q, M}^{n}\left(I^{n} M\right)=0$. It follows that $\alpha=0$.

Lemma 5.3. Let $K$ be a loosely $n$-tight commutative semisimple $F$-algebra. Assume that both $F$ and $K$ are $n$-admissible over $F$ and that $J_{n+1}(K / F)=$ $I^{n+1}(K / F)$. Let $\gamma \in H_{q}^{n} K$. Then there exists a finite 2-extension $E / F$ such that $i_{E \otimes_{F} K / K}(\gamma)=0$.

Proof. This follows from the last lemma by the argument to prove Lemma 3.6 .

We next show that, given suitable conditions, $n$-tight over $F$ implies $n$-taut over $F$.

Proposition 5.4. Let $K$ be an $n$-tight commutative semisimple $F$-algebra. Assume that both $F$ and $K$ are $n$-admissible over $F$ and that $J_{n+1}(K / F)=$ $I^{n+1}(K / F)$. Then $K$ is $n$-taut over $F$.

Proof. We first show that $i_{K / F}: H_{q}^{n} F \rightarrow H_{q}^{n} K$ is surjective. Let $\gamma \in H_{q}^{n} K$. By Lemma 5.3 above there exists a finite 2-extension $E / F$ such that $i_{E \otimes_{F} K / K}(\gamma)=$ 0 . In particular, there exist a finite 2-extension $E / F$ such that $i_{L / K}(\gamma)$ lies in the image of $i_{L / E}: H_{q}^{n} E \rightarrow H_{q}^{n} L$, where $L=E \otimes_{F} K$. By Corollary 4.11 and induction on $[E: F]$, we may futher assume that $E=F(\sqrt{d})$ is a quadratic extension of $F$. Since $I^{n+1}(K / F)=0$ by $n$-tightness, $s_{E / F}^{*}: I^{n}(L / E) \rightarrow I^{n}(K / F)$ is surjective by Corollary 4.10. By Proposition 5.1, it follows that the map $H_{q}^{n}(L / E) \rightarrow H_{q}^{n}(K / F)$ induced by $\operatorname{cor}_{E / F}$ is surjective. Hence we have the following commutative exact diagram:

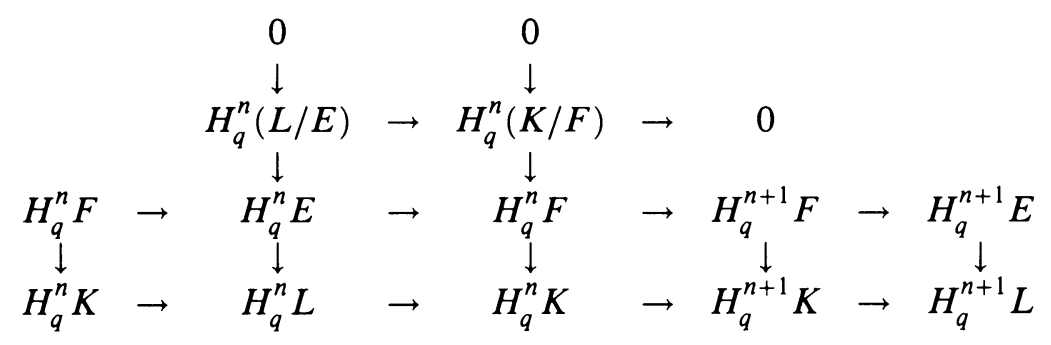

By chasing in this diagram, we see that, modulo the image of $i_{K / F}: H_{q}^{n} F \rightarrow$ $H_{q}^{n} K$, we may assume that $i_{L / K}(\gamma)=0$. Then $\gamma$ lies in $(d) \cup H_{q}^{n-1} K$ which lies in $e_{q, K}^{n}\left(I^{n} K\right)$ by condition (1) in the definition of $n$-admissibility. Now $i_{K / F}: I^{n} F \rightarrow I^{n} K$ is surjective by hypothesis. Consequently, $\gamma$ lies in the image of $i_{K / F}: H_{q}^{n} F \rightarrow H_{q}^{n} K$ as needed.

We next show that $i_{K / F}: H_{q}^{n+1} F \rightarrow H_{q}^{n+1} K$ is injective. Let $\alpha \in H_{q}^{n+1}(K / F)$. We must show that $\alpha=0$. There exists a finite 2-extension $E / F$ such that $i_{E / F}(\alpha)=0$. By Corollary 4.11 and induction on $[E: F]$, we may assume 
that $E=F(\sqrt{d})$ is a quadratic extension of $F$. Let $L=E \otimes_{F} K$. By the first part of the proof and Corollary 4.11, we have that $i_{L / E}: H_{q}^{n} E \rightarrow H_{q}^{n} L$ is surjective. Chasing in the above commutative exact diagram now shows $\alpha=0$ as needed.

Combining Corollary 4.11, Proposition 5.1, and Proposition 5.4, we get the following

Theorem 5.5. Let $K$ be an $n$-tight commutative semisimple $F$-algebra. Assume that both $F$ and $K$ are $n$-admissible over $F$ and that $J_{n+1}(K / F)=$ $I^{n+1}(K / F)(=0)$. Let $E / F$ be a finite 2-extension and $L=E \otimes_{F} K$. Then

(1) $L$ is $n$-tight over $E$ and $J_{n+1}(L / E)=I^{n+1}(L / E)$.

(2) $L$ is $n$-taut over $E$.

(3) $H_{q}^{n}(L / E)=e_{q, E}^{n}\left(I^{n}(L / E)\right)$.

One application of the above theorem is the following

Corollary 5.6. Let $K$ be a commutative semisimple $F$-algebra such that both $K$ and $F$ are n-admissible over $F$. Suppose that $i_{K / F}: I^{n} F \rightarrow I^{n} K$ is an isomorphism. Then $i_{K / F}: H_{q}^{m} F \rightarrow H_{q}^{m} K$ is an isomorphism for all $m \geq n$.

Proof. Clearly, $K$ satisfies the hypotheses of Theorem 5.5 and $I^{n}(K / F)=0$. The conclusion now follows using Theorem 5.5 and Theorem 3.9.

The analog of the 'Going down' result of Proposition 3.21 can now be established.

Proposition 5.7. Let $K$ be a commutative semisimple $F$-algebra. Suppose that both $K$ and $F$ are $n$-admissible over $F$ and $i_{K / F}: I^{n} F \rightarrow I^{n} K$ is surjective. Let $F^{+}=F(\sqrt{-1})$ and let $K^{+}=F^{+} \otimes_{F} K$. Suppose that $K^{+}$is $n$-tight over $F^{+}$and $J_{n+1}\left(K^{+} / F^{+}\right)=I^{n+1}\left(K^{+} / F^{+}\right)$, i.e., $J_{n+1}\left(K^{+} / F^{+}\right)=0$. Then $K$ is loosely $n$-tight over $F$ and $J_{n+1}(K / F)=I^{n+1}(K / F)$.

Proof. We must show that $J_{n+1}(K / F)=I^{n+1}(K / F)=2 I^{n}(K / F)$ and is torsionfree. Let $\phi \in J_{n+1}(K / F)$. Since $J_{n+1}\left(K^{+} / F^{+}\right)=0$, the $n$-admissibility of $F$ implies that $\phi$ lies in $2 I^{n} F$. Since $i_{K^{+} / F^{+}}: I^{n} F^{+} \rightarrow I^{n} K^{+}$is surjective, chasing in diagram (D) with $E=F^{+}$and $L=K^{+}$shows we may assume that $\phi$ lies in $2 I^{n}(K / F)$. It follows that $J_{n+1}(K / F)=I^{n+1}(K / F)=2 I^{n+1}(K / F)$. To show that $I^{n+1}(K / F)$ is torsion-free, it suffices to show that $I^{n+1}(K / F)$ has no nontrivial elements of order two. Suppose to the contrary that $0 \neq \phi$ lies in $I^{n+1}(K / F)$ but $2 \phi=0$. By the Hauptsatz of [AP], there exists a maximal $r \geq 0$ such that $\phi=2^{r} \psi$ for some $\psi \in I^{n}(K / F)$. By what we have already shown, $r \geq 1$. By [AEJ1, Corollary 2.5], there exists an integer $m$ such that $(-1)^{r+m} \cup e_{q, F}^{n}(\psi)=0$. Since $i_{K / F}(\psi)=0$ and $e_{q, F}^{n}$ is well-defined, we have $i_{K / F}\left(e_{q, F}^{n}(\psi)\right)=0$. In particular, $i_{K / F}\left((-1) \cup e_{q, F}^{n}(\psi)\right)=0$. By Theorem 5.5, 
$K^{+}$is $n$-taut over $F^{+}$. It follows by Theorem 3.24 that $H^{n+1}(K / F)$ is $(-1)$ torsion-free. This implies that $(-1) \cup e_{q, F}^{n}(\psi)=0$. From the exact sequence $(\Delta)$ and Theorem 5.5, we hence have $e_{q, F}^{n}(\psi)=\operatorname{cor}_{F^{+} / F}\left(e_{q, F}^{n}(\tau)\right)$ for some $\tau$ in $I^{n}\left(K^{+} / F^{+}\right)$. Thus $e_{q, F}^{n}\left(\psi-s_{F^{+} / F}^{*}(\tau)\right)=0$, i.e., we have $\psi-s_{F^{+} / F}^{*}(\tau)$ lies in $J_{n+1}(K / F)=I^{n+1}(K / F)=2 I^{n}(K / F)$. Consequently $2 \psi=4 \mu$ for some $\mu$ in $I^{n}(K / F)$, so $\phi=2^{r+1} \mu$. This contradicts the choice of $r$.

Proposition 4.9 and the above result immediately yield the following

Corollary 5.8. Let $K$ be a commutative semisimple F-algebra. Suppose that both $K$ and $F$ are n-admissible over $F$. Suppose that $i_{K / F}: I^{n} F \rightarrow I^{n} K$ is surjective. Let $F^{+}=F(\sqrt{-1})$ and $K^{+}=F^{+} \otimes_{F} K$. Then the following are equivalent:

(1) $J_{n+1}(K / F)=I^{n+1}(K / F)=2 I^{n+1}(K / F)$ and is torsion-free. $(=0)$.

(2) $i_{K^{+} / F^{+}}: I^{n} F^{+} \rightarrow I^{n} K^{+}$is surjective and $J_{n+1}\left(K^{+} / F^{+}\right)=I^{n+1}\left(K^{+} / F^{+}\right)$

The generalization of Theorem 5.5 to the loosely $n$-tight case is now easily established.

Theorem 5.9. Let $K$ be a loosely $n$-tight commutative semisimple $F$-algebra. Assume that both $F$ and $K$ are $n$-admissible over $F$ and that $J_{n+1}(K / F)=$ $I^{n+1}(K / F)$. Let $E / F$ be a finite 2-extension and let $L=E \otimes_{F} K$. Then

(1) $L$ is loosely $n$-tight over $E$ and $J_{n+1}(L / E)=I^{n+1}(L / E)$.

(2) $L$ is loosely $n$-taut over $E$.

(3) $H_{q}^{m}(L / E)=(-1)^{m-n} \cup e_{q, E}^{n}\left(I^{n}(L / E)\right)$ for all $m \geq n$.

Proof. We have already shown that (1) holds. Let $E^{+}=E(\sqrt{-1})$ and $L^{+}=$ $E^{+} \otimes_{E} L$. Since $L^{+}$is loosely $n$-tight over $E^{+}$by Proposition 4.9 and since $E^{+}$is not formally real, $L^{+}$is $n$-tight over $E^{+}$. By Theorem $5.5, L^{+}$is $n$-taut over $E^{+}$. Since $i_{L / E}: I^{n} E \rightarrow I^{n} L$ is surjective, it follows that the subgroup of $H_{q}^{n} L$ generated by $n$-fold cup products of elements in $H_{q}^{1} L$, viz., $e_{q, L}^{n}\left(I^{n} L\right)$ lies in the image of $i_{L / E}: H_{q}^{n} E \rightarrow H_{q}^{n} L$. By Proposition 3.17 and Remark 3.19, it follows that $L$ is loosely $n$-taut over $E$. This shows (2). Using Proposition 5.1 , statement (3) follows from (2) and Theorem 3.24.

We now come to the main result of this section.

Theorem 5.10. Let $K$ be a loosely $n$-tight commutative semisimple $F$-algebra. Assume that both $F$ and $K$ are $n$-admissible over $F$ and that $J_{n+1}(K / F)=$ $I^{n+1}(K / F)$. Then $\bar{e}_{q, F}^{m}$ is a well-defined isomorphism for all $m \geq n$ if $\bar{e}_{q, K}^{m}$ is a well-defined isomorphism for all $m \geq n$.

Proof. We first show that $e_{q, F}^{m}$ is well-defined for all $m \geq n$. By assumption this is true for $m=n$ so assume that $m>n$. Let $\tau_{i}$ be $m$-fold Pfister forms and $a_{i} \in \dot{F}$ for $1 \leq i \leq r$ satisfying $\sum_{i=1}^{r}\left\langle a_{i}\right\rangle \otimes \tau_{i}=0$. We must show that 
the element $\alpha:=\sum_{i=1}^{r} e_{q, F}^{m}\left(\tau_{i}\right)$ vanishes in $H_{q}^{n} F$. Since $e_{q, K}^{m}$ is well-defined, we have $i_{K / F}(\alpha)=0$. Thus $\alpha \in H_{q}^{m}(K / F)$. If $E$ is a euclidean field then $e_{q, E}^{m}$ is well-defined. In particular, $i_{E / F}(\alpha)=0$ for every euclidean closure $E$ of $F$. It follows by [A2, Satz 3] that $\alpha$ is a $(-1)$-torsion element in $H_{q}^{n} F$. By Theorem 5.9, we know that $K$ is loosely $n$-taut over $F$, so by Theorem 3.24 we get that $H_{q}^{m}(K / F)$ is $(-1)$-torsion-free. Consequently, $\alpha=0$.

Next we show that $e_{q, F}^{m}$ is surjective for all $m \geq n$. As noted before, $i_{K / F}: I^{n} F \rightarrow I^{n} K$ surjective implies that $i_{K / F}: I^{m} F \rightarrow I^{m} K$ is surjective for all $m \geq n$. By hypothesis $e_{q, K}^{m}$ is surjective for all $m \geq n$. Thus, to show our claim, we need only show that the image of $e_{q, F}^{m}$ contains $H_{q}^{m}(K / F)$. But this follows from Theorem 5.5.

To finish we must show that $\operatorname{ker}\left(e_{q, F}^{m}\right)=I^{m+1} F$ for all $m \geq n$. Since $i_{K / F}\left(\operatorname{ker}\left(e_{q, F}^{m}\right)\right) \subseteq \operatorname{ker}\left(e_{q, K}^{m}\right)=I^{m+1} K$ and, as noted above, $i_{K / F}: I^{m+1} F \rightarrow$ $I^{m+1} K$ is surjective, it suffices to show that $\operatorname{ker}\left(e_{q, F}^{m}\right) \cap I^{m}(K / F) \subseteq I^{m+1} F$, i.e., $J_{m+1}(K / F) \subseteq I^{m+1} F$. To establish this we show that $J_{m+1}(K / F) \subseteq$ $2^{m-n+1} I^{n}(K / F)$ for all $m \geq n$. If $m=n$ this is true by hypothesis, so we may assume that $m>n$. If $K$ is $n$-tight over $F$ then this is trivial, since both sides are 0 . Thus we may assume that $\sqrt{-1} \notin F$. Let $F^{+}=F(\sqrt{-1})$ and $K^{+}=F^{+} \otimes_{F} K$. Suppose that $\phi \in J_{m+1}(K / F)$. We must show that $\phi$ lies in $2^{m-n+1} I^{n}(K / F)$. By induction, we may assume that $\phi$ lies in $2^{m-n} I^{n}(K / F)$. Say $\phi=2^{m-n} \otimes \chi$ where $\chi$ lies in $I^{n}(K / F)$. Taking $e_{q, F}^{m}$ of this equation, we get $0=e_{q, F}^{m}(\phi)=(-1)^{m-n} \cup e_{q, F}^{n}(\chi)$. Thus $(-1) \cup e_{q, F}^{n}(\chi) \in H_{q}^{n+1}(K / F)$ is zero, since $H_{q}^{n+1}(K / F)$ is $(-1)$-torsion-free by Theorem 3.24. By Corollary 3.22 then $e_{q, F}^{n}(\chi)$ lies in the image of $\operatorname{cor}_{F^{+} / F}: H_{q}^{n}\left(K^{+} / F^{+}\right) \rightarrow H_{q}^{n}(K / F)$. Using Theorem 5.5 we see that there exists an element $\psi \in I^{n}\left(K^{+} / F^{+}\right)$such that $e_{q, F}^{n}(\chi)=\operatorname{cor}_{F^{+} / F}\left(e_{q, F^{+}}^{n}(\psi)\right)=e_{q, F}^{n}\left(s_{F^{+} / F}^{*}(\psi)\right)$. Thus $\chi-s_{F^{+} / F}^{*}(\psi)$ lies in $J_{n+1}(K / F)=2 I^{n}(K / F)$. Since $2 s_{F^{+} / F}^{*}(\psi)=0$ and $m>n$, it follows that $\phi=2^{m-n} \chi=2^{m-n}\left(\chi-s_{F^{+} / F}^{*}(\psi)\right)$ lies in $2^{m-n+1} I^{n}(K / F)$. This concludes the proof.

Remark 5.11. Our proof shows that under the hypotheses of this theorem, we also have that $K$ is loosely $m$-tight over $F$ for all $m \geq n$.

Corollary 5.12. Assume for any field extension $M$ of $F$ that $\bar{e}_{q, M}^{m}$ is well-defined for all $m \leq n$ and an isomorphism for all $m<n$. Let $K$ be a loosely $n$-tight commutative semisimple $F$-algebra. Assume that $J_{n+1}(K / F)=I^{n+1}(K / F)$. Then $\bar{e}_{q, F}^{*}$ is a well-defined isomorphism if $\bar{e}_{q, K}^{*}$ is.

Proof. This follows immediately from Theorem 5.10 and Proposition 4.7. 
Corollary 5.13. Let $K$ be a loosely 4-tight commutative semisimple $F$-algebra. Assume that $J_{5}(K / F)=I^{5}(K / F)$. Then $\bar{e}_{q, F}^{*}$ is a well-defined isomorphism if $\bar{e}_{q, K}^{*}$ is.

A special case of Theorem 5.10 is already interesting. This is the case that $K$ equals $F_{q}$ (or the direct product of finitely many copies of $F_{q}$ ). Then the hypotheses in the theorem, besides the $n$-admissibility of $F$, are $I^{n+1} F=$ $2 I^{n} F, I^{n+1} F$ is torsion-free, and $J_{n+1} F=I^{n+1} F$. In this case, we do not need the last hypothesis (cf. Theorem 1.1 and Corollary 1.2).

Proposition 5.14. Assume that $F$ is n-admissible. Assume furthermore that $I^{n+1} F=2 I^{n} F$ and is torsion-free. Let $E$ be a finite 2-extension of $F$. Then $I^{n+1} E=2 I^{n} E$ and is torsion-free. Furthermore, $\bar{e}_{q, E}^{m}$ is a well-defined isomorphism for all $m \geq n$. In particular, $H_{q}^{n+1} E=0$ if $E$ is not formally real.

Proof. Suppose first that $\sqrt{-1} \in F$. Then the hypothesis implies that $I^{n+1} F=$ 0 and hence $I^{n+1} E=0$ for any finite 2-extension $E$ of $F$ by [A1, Satz 3.6 or EL3, Theorem 6.3]. We show that $J_{n+1} F=0$. Let $\phi \in J_{n+1} F$. There exists a finite 2-extension $E$ of $F$ such that $i_{E / F}(\phi)=0$. By induction on $[E: F]$ we may assume that $E=F(\sqrt{d})$ is a quadratic extension of $F$. By hypothesis $I^{n} F \rightarrow J_{n+1} F \rightarrow J_{n+1} E$ is exact so $\phi \in\langle 1,-d\rangle \otimes I^{n} F \subseteq I^{n+1} F=0$. Thus $\phi=0$.

Suppose next that $\sqrt{-1} \notin F$. Let $F^{+}=F(\sqrt{-1})$. Since $I^{n+1} F=2 I^{n} F$ is torsion-free, $I^{n+1} F^{+}=0$ by [EP, Theorem 3.3]. Thus the argument above shows that $J_{n+1} F^{+}=0$ also. The exactness of the sequence $I^{n} F \rightarrow J_{n+1} F \rightarrow$ $J_{n+1} F^{+}$shows that $J_{n+1} F=2 I^{n} F \subseteq I^{n+1} F$. Thus, in all cases, we have $J_{n+1} F=2 I^{n} F=I^{n+1} F$.

Now let $K=F_{q}$. Then the hypotheses of Theorem 5.10 are satisfied. Let $E$ be a finite 2-extension of $F$. Then $L=E \otimes_{F} K$ is a direct product of finitely many copies of $F_{q}$. In particular, $\bar{e}_{q, L}^{m}$ is a well-defined isomorphism for all $m$. The conclusion of the proposition now follows by Proposition 4.9 and Theorem 5.10.

Corollary 5.15. Assume that $I^{5} F=2 I^{4} F$ and is torsion-free. Then $\bar{e}_{q, E}^{*}$ is a well-defined isomorphism for every 2-extension $E$ of $F$.

Proof. $F$ is 4 -admissible by Corollary 4.8. Since $\bar{e}_{q, E}^{n}$ is an isomorphism for $n \leq 3$, the result follows from the proposition above.

By Corollary 5.8, we can rephrase the corollary above as

Corollary 5.16. Assume that $I^{5} F(\sqrt{-1})=0$. Then $\bar{e}_{q, E}^{*}$ is a well-defined isomorphism for every 2-extension $E$ of $F$.

Of course, Theorem 1.1 can also be used to show this. Unfortunately, we have not been able to prove Theorems 5.9 or 5.10 without the extra hypothesis on $J_{n+1}$. 


\section{Abstract Witt Rings}

In this section, we shall apply the results of the previous sections to give a new proof of the main result in [AEJ2]. Indeed, we shall prove a much more general result.

We shall use the term abstract Witt ring in the sense of Marshall. For details see the book [Ma]. But we recall here:

An abstract Witt ring is a commutative ring $R$ together with a distinguished group of units in $R$ that generates $R$. This group of units is assumed to be an elementary abelian 2-group and to contain -1 . Its elements are called onedimensional forms.

There is a natural ring homomorphism $R \rightarrow \mathbf{Z} / 2 \mathrm{Z}$ mapping one-dimensional forms to 1 . The kernel of this homomorphism, the ideal of even-dimensional forms, is denoted by $I R$. We let $I^{n} R:=(I R)^{n}$.

A morphism of abstract Witt rings is a ring homomorphism that maps onedimensional forms to one-dimensional forms. The category of abstract Witt rings has arbitrary products. (Ring theoretically they are fiber products over $Z / 2 Z$.) If $R$ is an abstract Witt ring and $\Lambda$ an elementary abelian (multiplicative) 2-group then the group ring $R[\Lambda]$ is, in a natural way, an abstract Witt ring.

For convenience of expression, we make the following two definitions which are to be used in this section.

Definition 6.1. We say that a field $F$ is placid if $\bar{e}_{q, F}^{*}: G W F \rightarrow H_{q}^{*} F$ is a welldefined isomorphism. We say that a field $F$ is hereditarily placid if $\bar{e}_{q, E}^{*}: G W E$ $\rightarrow H_{q}^{*} E$ is a well-defined isomorphism for every finite 2-extension $E$ of $F$.

Definition 6.2. We say that an abstract Witt ring $R$ is docile if every field $F$ such that $W F \cong R$ (as abstract Witt rings) is placid. We say that $R$ is hereditarily docile if every field $F$ such that $W F \cong R$ is hereditarily placid.

Examples 6.3. (1) If $I^{5} R=2 I^{4} R$ and $I^{5} R$ is torsion-free then $R$ is hereditarily docile by Corollary 5.15. In particular, $\mathbf{Z} / 2 \mathbf{Z}, \mathbf{Z} / 4 \mathbf{Z}, \mathbf{Z}, \mathbf{Z} / 2 \mathbf{Z}[\xi]$ with $\xi^{2}=$ 1 , and $\mathbf{Z}[\xi]$ with $\xi^{2}=1$ are hereditarily docile.

(2) Every reduced abstract Witt ring of finite chain length is docile (cf. [ J, Theorems 5 and 6]).

For the first proposition in this section, we need a result that seems to be known but not explicitly stated anywhere. We state it as follows:

Lemma 6.4. Let $v$ be a 2-henselian valuation of $F$ and denote by $\bar{F}$ its residue class field. Then $F$ is placid if and only if $\bar{F}$ is placid.

Proof. Choose a subgroup $\Lambda$ of $\dot{F} / \dot{F}^{2}$ such that $v$ maps $\Lambda$ bijectively onto $\Gamma / 2 \Gamma$ where $\Gamma$ is the value group of $v$. Then there are natural descriptions of $W F$ (respectively, $H_{q}^{*} F$ ) in terms of $W \bar{F}$ (respectively, $H_{q}^{*} \bar{F}$ ) and $\Lambda$ (cf. [W]). The result immediately follows by comparing these descriptions. 
Proposition 6.5. Let $R$ be an abstract Witt ring and let $\Lambda$ be an elementary abelian 2-group. Then $R[\Lambda]$ is docile (respectively, hereditarily docile) if and only if $R$ is docile (respectively, hereditarily docile).

Proof. Let $v$ be a 2-henselian valuation of $F$ and denote by $\bar{F}$ its residue class field and by $\Gamma$ its value group. Then $W F \cong W \bar{F}[V]$ where $V \cong \Gamma / 2 \Gamma$. Using Lemma 6.4, it clearly follows that $R$ is docile (respectively, hereditarily docile) if $R[\Lambda]$ is docile (respectively, hereditarily docile). This also shows, that in proving the opposite implication, we may assume that $R$ is not a group ring (i.e., $R$ is basic). So assume that $W F \cong R[\Lambda]$. By [AEJ4, Theorem 4.6 and Remark 4.9(iii)], there is a 2-henselian valuation $v$ on $F$ such that $W \bar{F} \cong R$, where $\bar{F}$ is the residue class field, except possibly in the case $R=\mathrm{Z} / 2 \mathrm{Z}$ or $R=\mathbf{Z}$, where we might only get $W \bar{F} \cong \mathbf{Z} / 2 \mathbf{Z}[\xi]$ or $W \bar{F} \cong \mathbf{Z}[\xi]$ with $\xi^{2}=1$. In any case, $\bar{F}$ is placid (respectively, hereditarily placid). Hence the result follows by Lemma 6.4 .

Unfortunately, we have not been able to prove that direct products of docile Witt rings are docile. We do, however, have partial results.

Let $\sigma: W F \rightarrow R$ be a morphism of abstract Witt rings. We say that a field extension $K$ of $F$ realizes $\sigma$ if there is an isomorphism $\tau: W K \rightarrow R$ of abstract Witt rings such that $\sigma$ corresponds to $i_{K / F}: W F \rightarrow W K$, i.e., $\sigma=$ $\tau \circ i_{K / F}$. In the case we are given an isomorphism $W F \cong R \times S$ and $\sigma: W F \rightarrow R$ is the morphism corresponding to the projection $R \times S \rightarrow R$, we shall simply say that $K$ realizes the factor $R$ of $W F$. Conversely, when we write $W F \cong$ $W K \times S$, we mean to imply that the morphism $W F \rightarrow W K$ corresponding to the projection $W K \times S \rightarrow W K$ is $i_{K / F}$.

Lemma 6.6. Suppose that we have an isomorphism $W F \cong R_{1} \times \cdots \times R_{k}$ of abstract Witt rings. Assume that for each $i$ there exists a field extension $K_{i}$ of $F$ realizing the factor $R_{i}$ of $F$. Then $F$ is placid (respectively, hereditarily placid) if $K_{1}, \ldots, K_{k}$ are all placid (respectively, hereditarily placid).

Proof. Let $K=\prod_{i} K_{i}$. Then $K$ is 1-tight over $F$. Let $E$ be a finite 2extension of $F$ and let $L=E \otimes_{F} K$. Then $L=\prod_{j} L_{j}$ where each $L_{j}$ is a finite 2-extension of some $K_{i}$. By Corollary 4.13, we know that $L$ is 1-tight over $E$. In particular, $E$ is placid if each $L_{j}$ is placid by Corollary 5.12. The result follows.

We do not know if factors of Witt rings of fields are always realizable. If we knew this, it would follow from the lemma above the direct products of docile abstract Witt rings were docile. In [AEJ4, Realization Theorem 4.8], however, we proved that every factor of $W F$ that is a nontrivial group ring is realizable. Using Proposition 6.5, we hence get the following

Corollary 6.7. A finite direct product of nontrivial group rings over docile (respectively, hereditarily docile) abstract Witt rings is docile (respectively, hereditarily docile). 
The following lemma gives a sufficient condition for a field $K$ to realize a factor of $W F$.

Lemma 6.8. Suppose that we have an isomorphism $W F \cong R \times S$ of abstract Witt rings and let $\sigma: W F \rightarrow R$ be the morphism corresponding to the projection $R \times S \rightarrow R$. Let $K$ be a field extension of $F$ and let $\tau: W K \rightarrow R$ be a morphism of abstract Witt rings such that $\sigma=\tau \circ i_{K / F}$. Assume that $\tau$ is injective on onedimensional forms. Then $\tau$ is an isomorphism, i.e., $K$ realizes the factor $R$ of $W F$.

Proof. From the definition of $\sigma$, it follows that $\sigma$ maps the one-dimensional forms in $W F$ surjectively onto the one-dimensional forms in $R$. As $\tau$ is injective on one-dimensional forms, it follows that $\tau$ is an isomorphism on onedimensional forms and that $i_{K / F}$ is surjective on one-dimensional forms, hence surjective. In particular, $\operatorname{ker}(\tau)=i_{K / F}(\operatorname{ker}(\sigma))$. From the definition of $\sigma$, it follows easily that $\operatorname{ker}(\sigma)$ is generated by differences of two one-dimensional forms. Using the hypothesis on $\tau$ again, it follows that $\operatorname{ker}(\tau)=0$. Clearly, $\tau$ is surjective.

The next lemma says that if several factors of $W F$ can be realized individually, then their direct product is also realizable.

Lemma 6.9. Suppose that we have an isomorphism $W F \cong R_{1} \times \cdots \times R_{k} \times S$ of abstract Witt rings. Assume that for each $i$ there exists a field extension $K_{i}$ of $F$ realizing the factor $R_{i}$ of $W F$. Then the factor $R=R_{1} \times \cdots \times R_{k}$ of $W F$ is realized by a field extension $K$ of $F$. Moreover, if each $K_{i}$ is placid (respectively, hereditarily placid) then $K$ can be chosen to be placid (respectively, hereditarily placid).

Proof. Let $K_{i}^{0}$ be the quadratic closure of $F$ in $K_{i}$. Then $W K_{i}^{0} \rightarrow R_{i}$ is injective on one-dimensional forms and hence is an isomorphism by Lemma 6.8. In particular, $K_{i}$ is 1-tight over $K_{i}^{0}$. So, by Corollary 5.12 (and Corollary 4.13), we have $K_{i}^{0}$ placid (respectively, hereditarily placid) if $K_{i}$ is placid (respectively, hereditarily placid). This shows that, replacing $K_{i}$ by $K_{i}^{0}$, we may assume that each $K_{i}$ is a 2-extension of $F$. But then we clearly also may assume that each $K_{i} \subseteq F_{q}$. Now let $K=K_{1} \cap \cdots \cap K_{k}$. Then $W K \rightarrow R_{1} \times$ $\cdots \times R_{k}$ is injective on one-dimensional forms and, therefore, an isomorphism by Lemma 6.8. The result now follows by Lemma 6.6.

Before we state the main results of this section, we introduce some terminology.

Let $\mathscr{B}$ be a class of abstract Witt rings. By the class $\mathscr{C}_{\mathscr{B}}$ generated by $\mathscr{B}$, we mean the smallest class of abstract Witt rings that contains $\mathscr{B}$ and is closed under the following operations:

(1) taking an isomorphic abstract Witt ring,

(2) taking a finite product of abstract Witt rings, 
(3) taking a group Witt ring of an abstract Witt ring.

Of course, every Witt ring in $\mathscr{C}_{\mathscr{B}}$ can be constructed in a finite number of steps out of Witt rings in $\mathscr{B}$ using these operations. We use this to define a filtration $\mathscr{B}_{0} \subseteq \mathscr{B}_{1} \subseteq \cdots$ of $\mathscr{C}_{\mathscr{B}}$ as follows:

$R$ is in $\mathscr{B}_{0}$ if $R$ is isomorphic to a finite product of abstract Witt rings in the class $\mathscr{B}$.

$R$ is in $\mathscr{B}_{k+1}$ whenever $R$ is isomorphic to a finite product of group rings over Witt rings in $\mathscr{B}_{k}$.

Clearly, every object in $\mathscr{B}_{k+1}$ is isomorphic to a finite direct product of abstract Witt rings that are either nontrivial group rings over objects in $\mathscr{B}_{k}$ or are in $\mathscr{B}$.

A possible method to prove that every Witt ring $R$ in $\mathscr{C}_{\mathscr{B}}$ is docile is to use induction on the degree of $R$ with respect to this filtration. Let us, for simplicity, assume that $\mathscr{B}$ is closed under taking finite products of abstract Witt rings. Then, in the initial step, we are given that $W F \cong S$ with $S$ in $\mathscr{B}$ and have to show that $F$ is placid. (This is the absolute case.) In the induction step, we are given that $W F$ is isomorphic to a finite product $R_{1}\left[\Lambda_{1}\right] \times \cdots \times R_{r}\left[\Lambda_{r}\right] \times S$ of abstract Witt rings where each $R_{i}$ is docile, each $\Lambda_{i}$ is nontrivial, and $S$ is in $\mathscr{B}$. Then, by Proposition 6.5 and [AEJ4, Realization Theorem 4.8], each factor $R_{i}\left[\Lambda_{i}\right]$ is docile and realizable. Hence, by Corollary 6.7 and Lemma 6.9 the factor $R=R_{1}\left[\Lambda_{1}\right] \times \cdots \times R_{r}\left[\Lambda_{r}\right]$ of $W F$ is docile and realizable. So we have that $W F \cong W K \times S$ where $K$ is a placid field extension of $F$ and $S$ is in $\mathscr{B}$ and have to prove that $F$ is placid. (This is the relative case.) Note that the relative case contains the absolute case by letting $K=F_{q}$. The same reasoning works for hereditarily docile instead of docile. We state the result of this discussion as a proposition.

Proposition 6.10. Let $\mathscr{B}$ be a class of abstract Witt rings. Assume that for every field $F$ the following holds:

If there exists a placid (respectively, hereditarily placid) field extension $K$ of $F$ and abstract Witt rings $S_{1}, \ldots, S_{s}$ in $\mathscr{B}$ such that $W F \cong W K \times S_{1} \times \cdots \times S_{s}$ then $F$ is placid (respectively, hereditarily placid).

Then every abstract Witt ring in the class $\mathscr{C}_{\mathscr{B}}$ is docile ( respectively, hereditarily docile).

Assume that we have $W F \cong W K \times S$ as in the discussion above. Then $i_{K / F}: W F \rightarrow W K$ is surjective and has kernel isomorphic to $I S$. In particular, if $S$ is 'small' then $i_{K / F}: W F \rightarrow W K$ is 'not far from being an isomorphism'. This is the connection to the preceding sections. We now come to the applications.

Theorem 6.11. Assume for every field $F$ that $\bar{e}_{q, F}^{m}$ is a well-defined isomorphism for all $m<n$ and a well-defined monomorphism for $m=n$. Let $\mathscr{B}$ be the class 
of abstract Witt rings $R$ such that $I^{n+1} R=2 I^{n} R$ and $I^{n+1} R$ is torsion-free. Then every Witt ring in the class $\mathscr{C}_{\mathscr{B}}$ generated by $\mathscr{B}$ is hereditarily docile.

Proof. We shall use the proposition above. As $\mathscr{B}$ is clearly closed under taking finite direct products, we may assume that we have given that $W F \cong W K \times$ $S$ with a hereditarily placid field extension $K$ of $F$ and $S$ in $\mathscr{B}$. This implies that $i_{K / F}: I^{m} F \rightarrow I^{m} K$ is surjective and has kernel isomorphic to $I^{m} S$ for every $m \geq 1$. We have to show that $F$ is hereditarily placid. By the description of $\mathscr{B}$, we have that $K$ is loosely $n$-tight over $F$. By Proposition 4.7, our hypothesis implies that every commutative semisimple $F$-algebra is $n$-admissible. Furthermore, our hypothesis implies that $J_{n+1} F=I^{n+1} F$, hence $J_{n+1}(K / F)=I^{n+1}(K / F)$. Now let $E$ be a finite 2-extension of $F$ and let $L:=E \otimes_{F} K$. By Theorem 5.9, we know that $L$ is loosely $n$-tight over $E$ and $J_{n+1}(L / E)=I^{n+1}(L / E)$. As $K$ is hereditarily placid $\bar{e}_{q, L}^{*}$ is a well-defined isomorphism. Hence, by Corollary 5.12 , we have that $E$ is placid.

Corollary 6.12. Let $\mathscr{B}$ be the class of abstract Witt rings $R$ such that $I^{4} R=$ $2 I^{3} R$ and $I^{4} R$ is torsion-free. Then every Witt ring in the class $\mathscr{C}_{\mathscr{B}}$ generated by $\mathscr{B}$ is hereditarily docile.

We need the notion of Milnor $k$-theory for abstract Witt rings.

Definition 6.13. Let $R$ be an abstract Witt ring. We denote by $k_{*} R$ the graded $\mathrm{Z} / 2 \mathrm{Z}$-algebra generated by symbols $l(a)$ in degree one, where $a$ runs through the one-dimensional forms in $R$, subject only to the defining relations $l(a \cdot b)=$ $l(a)+l(b)$ and $l(a) l(b)=0$ whenever $(1-a) \cdot(1-b)=0$. If $S$ is another abstract Witt ring we define the product $k_{*} R \times k_{*} S$ to be $k_{*}(R \times S)$.

If $R=W F$ then $k_{*} R$ is the Milnor $K$-ring $k_{*} F$ (cf. [Mi]). In this case we have a natural morphism of graded rings $h_{F}^{*}: k_{*} F \rightarrow H_{q}^{*} F$ induced by $l\left(a_{1}\right) \cdots l\left(a_{n}\right) \mapsto\left(a_{1}\right) \cup \cdots \cup\left(a_{n}\right)$. If $n \leq 3$ then $h_{F}^{n}: k_{n} F \rightarrow H_{q}^{n} F$ is an isomorphism (cf. [Mi] for $n=1$, [Me] for $n=2$, [MS or R] for $n=3$ ).

Theorem 6.14. Assume for every field $F$ that the homomorphisms $\bar{e}_{q, F}^{m}$ and $h_{F}^{m}$ are well-defined isomorphisms for all $m<n$ and well-defined monomorphism for $m=n$. Let $\mathscr{B}$ be the class of abstract Witt rings $R$ such that $I^{n+1} R=2 I^{n} R$ and $I^{n+1} R$ is torsion-free. Then $h_{F}^{*}$ is an isomorphism for every $F$ such that $W F$ lies in $\mathscr{C}_{\mathscr{B}}$.

Proof. As above, we reduce to the case that $W F \cong W K \times S$ with $K$ an extension field of $F$ such that $h_{K}^{*}$ is an isomorphism and $S$ lies in $\mathscr{B}$. Then it is easy to see that $k_{*} F \cong k_{*} K \times k_{*} S$ with all isomorphisms compatible with the maps induced by $F \subseteq K$. In particular, $\operatorname{ker}\left(k_{n+1} F \rightarrow H_{q}^{n+1} F\right)$ is contained in $k_{n+1} S$. As $e_{q, E}^{*}$ is surjective for every finite 2-extension $E$ of $F$, we clearly have that $h_{E}^{*}$ is surjective for every such $E$. We show $h_{F}^{n+1}$ is injective. It follows from [EL1, Main Theorem 3.2] that $k_{n+1} S=l(-1) k_{n} S$. Let 
$F^{+}=F(\sqrt{-1})$. We have a commutative diagram

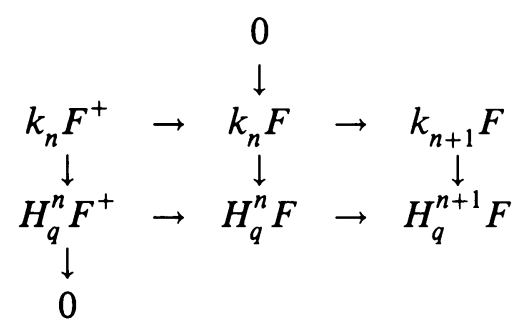

with exact columns and bottom row. Here the map $k_{n} F^{+} \rightarrow k_{n} F$ is induced by the norm and $k_{n} F \rightarrow k_{n+1} F$ is multiplication by $l(-1)$. Since $\operatorname{ker}\left(h_{F}^{n+1}\right) \subseteq$ $\operatorname{im}\left(k_{n} F \rightarrow k_{n+1} F\right)$, chasing in this diagram shows that $h_{F}^{n+1}$ is injective. Using induction, this argument can be used to show that $h_{F}^{m}$ is injective for all $m>$ $n$.

This theorem together with [MS or R] immediately yields the following

Corollary 6.15. Let $\mathscr{B}$ be the class of abstract Witt rings $R$ such that $I^{4} R=$ $2 I^{3} R$ and $I^{4} R$ is torsion-free. Then $h_{F}^{*}$ is an isomorphism for every $F$ such that $W F$ lies in $\mathscr{C}_{\mathscr{B}}$.

Using a slightly smaller class $\mathscr{B}$ in Theorem 6.11 , we get by with a somewhat weaker hypothesis.

Theorem 6.16. Assume for every field $F$ that $\bar{e}_{q, F}^{m}$ is a well-defined isomorphism for all $m<n$ and well-defined for $m=n$. Let $\mathscr{B}$ be the class $\mathscr{C}_{\mathscr{B}}$ of abstract Witt rings $R$ such that

(1) $I^{n+1} R=2 I^{n} R$ and is torsion-free.

(2) Every class in $I^{n} R / I^{n+1} R$ is the class of an element of the type $(1-d) \cdot \tau$ where $d$ is a one-dimensional form and $\tau \in I^{n-1} R$.

Then every Witt ring in the class generated by $\mathscr{B}$ is hereditarily docile.

Proof. We can use the same proof as for Theorem 6.11, except for the part that $J_{n+1}(K / F)=I^{n+1}(K / F)$. We clearly only have to show that $J_{n+1}(K / F) \subseteq$ $I^{n+1} F$. So let $\phi \in J_{n+1}(K / F)$ be given. We see from the description of $\mathscr{B}$ that, modulo $I^{n+1}(K / F) \cong I^{n+1} S$, we may assume that $\phi=\langle 1,-d\rangle \otimes \psi$ for some $\psi$ in $I^{n-1}(K / F) \cong I^{n-1} S$. By the $n$-admissibility of $F$ the hypothesis of Proposition 2.7 holds so $\phi$ lies in $I^{n+1} F$ as needed.

The present state of knowledge about the $e_{q, F}^{m}$ in general now gives the following

Corollary 6.17. Let $\mathscr{B}$ be the class of abstract Witt rings $R$ such that

(1) $I^{5} R=2 I^{4} R$ and is torsion-free.

(2) Every class in $I^{4} R / I^{5} R$ is the class of an element $(1-d) \cdot \tau$ for some one-dimensional form $d$ and some $\tau \in I^{3} R$. 
Then every Witt ring in the class $\mathscr{C}_{\mathscr{B}}$ generated by $\mathscr{B}$ is hereditarily docile.

Note that the class $\mathscr{B}$ in this theorem contains the Witt rings of $C_{4}$-fields and fields $F$ such that $F(\sqrt{-1})$ has 2-cohomological dimension at most 3 . The former type includes the Witt rings of function fields of transcendence degree $\leq 4$ over algebraically closed fields and the Witt rings of function fields of transcendence degree $\leq 3$ over finite fields. The latter type includes the Witt rings of function fields of transcendence degree $\leq 3$ over real closed fields and the Witt rings of function fields of transcendence degree $\leq 1$ over local or global fields. In particular, $\mathscr{C}_{\mathscr{B}}$ contains all Witt rings of elementary type (cf. [AEJ2, Corollary 4.4]).

\section{REFERENCES}

[A1] J. K. Arason, Cohomologische Invarianten quadratischer Formen, J. Algebra 36 (1975), 448491.

[A2] _ Primideale im graduierten Wittring und im $\bmod 2$ Cohomologiering, Math. Z. 145 (1975), 139-143.

[A3] _ , A proof of Merkurjev's Theorem, Canad. Math. Soc. Conf. Proc., vol. 4, Amer. Math. Soc., Providence, R.I., 1984, pp. 121-130.

[A4] _ , unpublished.

[AEJ1] _ , The graded Witt ring and Galois cohomology. I, Canad. Math. Soc. Conf. Proc., vol. 4, Amer. Math. Soc., Providence, R.I., 1984, pp. 17-50.

[AEJ2] J. K. Arason, R. Elman, and B. Jacob, Graded Witt rings of elementary type, Math. Ann. 272 (1985), 267-280.

[AEJ3] _ Fields of cohomological 2-dimension three, Math. Ann. 274 (1986), 649-657.

[AEJ4] _ _ Rigid elements, valuations, and realization of Witt rings, J. Algebra 110 (1987), 449467.

[AP] J. K. Arason and A. Pfister, Beweiss des Krullschen Durschnittsatzes für den Wittring, Invent. Math. 12 (1971), 173-176.

[Br] K. S. Brown, Cohomology of groups, Graduate Texts in Math., vol. 45, Springer-Verlag, Berlin and New York, 1982.

[Cr] T. Craven, Existence of SAP extension fields, Arch. Math. 29 (1977), 594-597.

[EL1] R. Elman and T. Y. Lam, Pfister forms and K-theory of fields, J. Algebra 23 (1972), 181-213.

[EL2] _ Q Quadratic forms over formally real fields and pythagorean fields, Amer. J. Math. 23 (1972), 1155-1194.

[EL3] __, Quadratic forms under algebraic extensions, Math. Ann. 219 (1976), 21-42.

[EP] R. Elman and A. Prestel, Reduced stability of the Witt ring of a field and its pythagorean closure, Amer. J. Math. 106 (1984), 1237-1260.

[J] B. Jacob, On the structure of Pythagorean fields, J. Algebra 68 (1981), 247-267.

[JR] B. Jacob and M. Rost, Degree four cohomological invariants for quadratic forms, Invent. Math. (to appear).

[K] M. Knebusch, Generic splitting of quadratic forms. I, Proc. London Math. Soc. (3) 33 (1976), 65-93.

[L] T. Y. Lam, The algebraic theory of quadratic forms, Benjamin, New York, 1973.

[Ma] M. Marshall, Abstract Witt rings, Queen's Papers in Pure and Applied Mathematics, no. 57, 1980.

[Me] A. S. Merkurjev, On the norm residue symbol of degree 2, Dokl. Akad. Nauk SSSR 261 (1981), 542-547; English transl., Soviet Math. Dokl. 24 (1981), 546-551. 
[MS] A. S. Merkurjev and A. A. Suslin, On the norm residue homomorphism of degree three, preprint. [Mi] J. Milnor, Algebraic K-theory and quadratic forms, Invent. Math. 9 (1970), 318-344.

[P] A. Pfister, Quadratische Formen in beliebigen Körpern, Invent. Math. 1 (1966), 116-132.

[R] M. Rost, Hilbert 90 for $K_{3}$ for degree two extensions, preprint.

[S] J.-P. Serre, Cohomologie Galoisienne, Lecture Notes in Math., vol. 5, Springer-Verlag, Berlin and New York, 1965.

[Sch] W. Scharlau, Quadratic and Hermitian forms, Springer-Verlag, Berlin and New York, 1985.

[T] J. Tate, Duality theorems in Galois cohomology over number fields, Proc. Internat. Congr. Math. Stockholm (1963), 288-295.

[W] A. Wadsworth, p-Henselian fields: K-theory, Galois cohomology, and graded Witt rings, Pacific J. Math. 105 (1983), 473-495.

Raunvisindastofnun Haskolans, University of Iceland, Reykjavik, Iceland (Current address of J. K. Arason)

Department of Mathematics, University of California at Los Angeles, Los Angeles, CALIFORNIA 90024 (Current address of Richard Elman)

Department of Mathematics, Oregon State University, Corvallis, Oregon 97333

Current address (Bill Jacob): Department of Mathematics, University of California, Santa Barbara, California 93106 